\title{
Long-term starspot evolution, activity cycle and orbital period variation of SZ Piscium
}

\author{
A. F. Lanza ${ }^{1}$, M. Rodonò $\grave{1}^{1,2}$, L. Mazzola $^{2}$, and S. Messina ${ }^{1}$ \\ 1 Osservatorio Astrofisico di Catania, Italy \\ e-mail: sme@sunct.ct.astro.it \\ 2 Dip. di Fisica e Astronomia, Università degli Studi, Via Santa Sofia, 78, Città Universitaria, \\ 95125 Catania, Italy \\ e-mail: mrodono@ct.astro.it; lma@sunct.ct.astro.it
}

Received 4 May 2001 / Accepted 11 July 2001

\begin{abstract}
A sequence of $V$-band light curves of the active close binary SZ Piscium (F8 V-IV+ K1 IV), extending from 1957 to 1998 , is presented and analysed to derive the spot distribution and evolution on the component stars. In our modelling approach the Roche geometry and Kurucz's atmospheric models were adopted. The resulting maps of the spot surface distribution were regularized by means of the Maximum Entropy and Tikhonov criteria to take full advantage of the increased geometrical resolution during eclipses. By comparing the maps obtained with these two criteria, it was possible to discriminate between surface features actually required by the data and artifacts introduced by the regularization process. Satisfactory fits were obtained assuming spots on both components and the unspotted $V$-band luminosity ratio: $L_{1} / L_{2}=0.35 \pm 0.035$. The derived yearly spot distributions indicate that the spot patterns consist of two components, one uniformly and the other non-uniformly distributed in longitude, the latter suggesting the presence of preferential longitudes. Starspots at latitudes higher than $\sim 75^{\circ}$ were not required to reproduce the photometric modulation. For the spot pattern on the less luminous F8 V primary component there are only some hints of the possible presence of spots around the substellar point facing the K1IV secondary component. On the more luminous and larger secondary component there is clear evidence for the presence of three active longitudes. A stable active longitude around the substellar point facing the primary star appears to be quite compact with an extension of $\sim 40^{\circ}$ on several maps. The other two active longitudes display irregular changes of the spot area and location. The variation of the total spotted area on the secondary component shows sizeable fluctuations and a possible short-term cycle of about $13 \mathrm{yr}$, which is more clearly apparent in the variation of the spotted area unevenly distributed in longitude. A longer-term cycle of about $30 \mathrm{yr}$ with a markedly non-sinusoidal variation might also be present. The spatial association between photospheric spots and chromospheric plages (as detected in the UV lines) appears significant for the active region around the substellar point in the K1 IV component. A possible connection between magnetic activity and orbital period variation is suggested by comparing the variation of the total spotted area with the $\mathrm{O}-\mathrm{C}$ diagram of the primary eclipse times. The time span covered by the available data is, however, not long enough for us to draw any definite conclusions.
\end{abstract}

Key words. stars: activity - binaries: close - binaries: eclipsing - stars: individual (SZ Piscium) - starspots

\section{Introduction}

SZ Piscium (HD 219113; SAO 128041; BD+01 4695) is an eclipsing binary (F8 V-IV+K1 IV) belonging to the RS Canum Venaticorum class of variable stars (Hall 1976). They are characterized by intense solar-like magnetic activity, with typical energy scales two or three orders of magnitude larger than in the Sun (Guinan \& Gimenez 1993; Strassmeier et al. 1993; and reference therein).

Send offprint requests to: A. F. Lanza,

e-mail: nla@sunct.ct.astro.it
The present study is framed in the context of the solarstellar connection, i.e., we adopt the observed structure and phenomena of the solar atmosphere as a paradigm to interpret stellar observations. SZ Piscium is well suited for such a kind of approach because it is among the most active and well observed RS CVn binaries showing a strong chromospheric emission in the Ca II H\&K and $\mathrm{H} \alpha$ lines of the K1 IV component (Jakate et al. 1976; Popper 1988; Doyle et al. 1994a; Frasca \& Catalano 1994; Fernandez-Figueroa et al. 1994). It was detected also as a X-ray, EUV and radio source with remarkable time 
variability (e.g., Dempsey et al. 1993; Pye et al. 1995; Mitrou et al. 1997; Doyle et al. 1994a; Florkowski et al. 1985). Flares have been observed not only in the chromospheric and in the EUV lines, but also in the optical band (Doyle et al. 1994a,b). A spectroscopic study of the variability of the $\mathrm{H} \alpha$ line intensity and profile was performed by Huenemoerder \& Ramsey (1984). They suggested the presence of active longitudes on the K1 IV secondary and possibly of circumstellar matter. Doyle et al. (1994a) found evidence for chromospheric inhomogeneities in the K1 IV star by looking at the variation of the Mg II $\mathrm{h} \& \mathrm{k}$ and other chromospheric lines versus phase. Popper (1988) presented a detailed spectroscopic study of the system deriving luminosities, radii and masses and found that both components are detached from the respective Roche lobes.

Optical light curves show a distintive modulation outside eclipse attributed to cool starspots that are regarded as stellar counterparts of sunspots or sunspot groups (Eaton \& Hall 1979). By modelling their surface distribution and their time variations it is possible to derive information on active longitudes and activity cycles. Eclipsing systems are particularly suited for this purpose because the knowledge of stellar parameters reduces the uncertainties of the spot modelling procedures based on photometric data alone. Moreover, during eclipses the geometric resolution is increased by the scanning effect produced by the transit of the occulting component over the disk of the eclipsed component, which allows us to perform an eclipse mapping of the distribution of the spots on the disk of the occulted star (cf. Cameron \& Hilditch 1997; Lanza et al. 1998a).

In the present paper we analyse a sequence of light curves of SZ Psc, consisting of the data available in the literature complemented with new observations collected between 1993 and 1998 by the Automated Photometric Telescope (APT-80) of Catania Observatory. Our modelling approach is based on the method described by Lanza et al. (1998a) and improved by using a more realistic atmospheric treatment. The application of the solar-stellar connection paradigm in the interpretation of the results is particularly fruitful when an extended sequence of photometric data is available, allowing us to address the study of active longitudes, activity cycles and surface differential rotation (cf. Rodonò \& Lanza 1996; Lanza \& Rodonò 1999a).

The orbital period of SZ Psc is not constant, as observed in several close binaries with a magnetically active component, and its variation was recently discussed by Kalimeris et al. (1995), who also considered some possible physical mechanisms responsible for the observed changes. Moreover, the study of the long-term activity of SZ Psc allows us a further test of the revised Applegate's (1992) model proposed by Lanza et al. (1998b) and Lanza \& Rodonò (1999b) on the connection between stellar hydromagnetic dynamos and orbital period variation in active close binaries.

\section{Observations}

Our data set consists of twenty yearly light curves of SZ Psc obtained from 1957 to 1998. Photometric data from 1957 to 1991 were taken from the literature, whereas data from 1993 to 1998 were obtained by the Automated Photometric Telescope (APT-80) of Catania Observatory on Mt. Etna. It is a Ritchey-Chrètien reflector with an aperture of $80 \mathrm{~cm}, \mathrm{f} / 8$, equipped with an RCA 1P21 photomultiplier and standard $U B V$ filters. The standard observing sequence is: $c-c k 1-c k 2-c-v-v-v-c-v-v-v-c$, where $c$ is the comparison star (HD 219018), $c k 1$ and $c k 2$ are the two check stars (HD 217459 and HD 218527, respectively) and $v$ is the variable star. References on the observing procedure and data reduction may be found in, e.g., Messina et al. (1999).

In the present analysis we consider only the $V$ band light curves of SZ Psc because they are the most complete and homogeneous. In addition to the standard correction for atmospheric extinction, all our differential magnitudes are referred to HD 219018, the mostly adopted comparison star. Its constancy was questioned by Jakate (1979). Subsequent observations proved it to be constant and a suitable comparison star (Eaton et al. 1982; Percy \& Welch 1982). The mean standard error of the observations is between 0.005 and $0.03 \mathrm{mag}$.

In Table 1 we give for each light curve the mid epoch of the observations, the extent $\Delta T$ over which photometric data were collected, the number of normal points $(M)$, the aperture of the telescope used $\left(D_{\mathrm{T}}\right)$, the photometric system ( $I=$ instrumental, $S=$ standard $U B V$ ), the ephemeris adopted to compute the phases of the light curve and the literature reference. In addition to the listed light curves, there are also some sparse photometric observations collected in 1967, 1982, 1983 and late 1996, which were disregarded because of their poor phase coverage.

Since the orbital period is very close to four days, it is difficult to obtain a good light curve coverage from a single observing site in a short time. Therefore, data points which appear rather close in phase could have been obtained several weeks or months apart, so that the system might have undergone significant variations. Actually, SZ Psc is a very active system and out-of-eclipse light variations up to 0.03-0.05 mag have been observed during the course of the same night. Such a short-term intrinsic variability is probably due to flares (cf., e.g., Doyle et al. 1994a). Jakate et al. (1976) and Eaton et al. (1982) also reported light changes with an amplitude of $\sim 0.05 \mathrm{mag}$ on time scales as short as the orbital period. These are probably related to short-term changes in the area and surface distribution of the spots. Due to the intrinsic variability and the unfavourable orbital period, most of the light curves show both phase gaps and large dispersion of the observations with intrinsic standard deviations up to 0.03-0.05 mag. In order to avoid a loss of information by averaging such variations, whenever possible the observations were not averaged into normal points. All points 


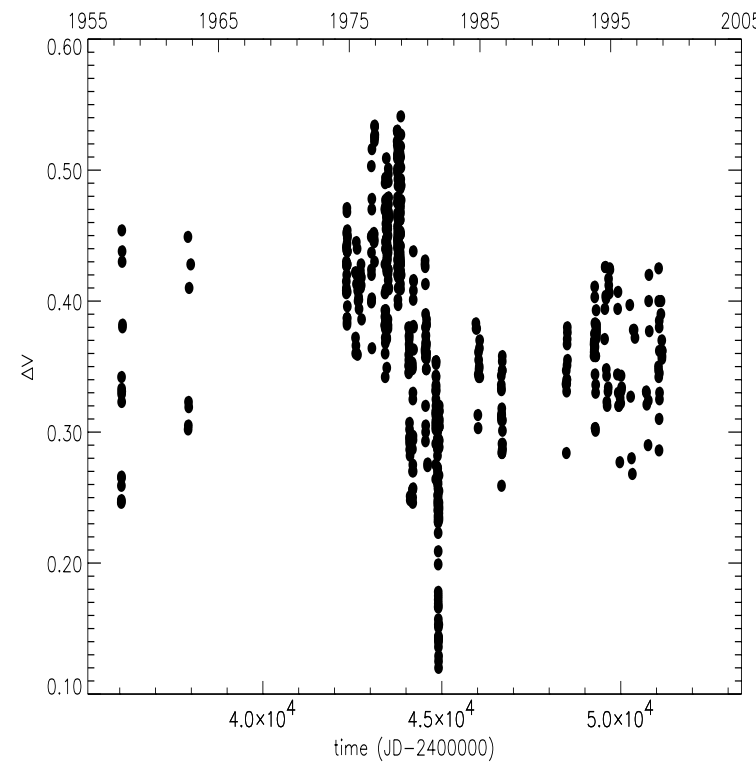

Fig. 1. The magnitude difference $\Delta V$ between the comparison HD 219018 and SZ Psc versus time. Only the photometric points outside eclipses are plotted.

in the light curves were given the same unit weight as in the analysis made by Lanza et al. (1998a).

The brightest magnitude from our light curve sequence turned out to be $m_{V}=7.155 \pm 0.005$ at orbital phase 0.6975 in the 1978 light curve. This value is assumed as the magnitude of the unspotted system at phase 0.6975 , thus establishing the magnitude scale calibration for the subsequent light curve modelling.

The overall variation of the system magnitude versus time was plotted in Fig. 1. In order to better display the long-term variation due to spot activity, only photometric points outside eclipses were plotted.

\section{Spot modelling technique}

The reconstruction of the intensity distribution over the surface of a star using photometric data alone is an illposed problem. The wide-band flux modulation provides us with information only on the variation of the projected spot area versus phase, i.e., the stellar longitude. Some additional information on the latitudinal distribution can be extracted during eclipses, when the spot pattern on the component being occulted is scanned by the disk of the other component. Nevertheless, the problem remains ill-posed even in the most favourable conditions.

We notice that one can select a unique spot distribution by minimizing the $\chi^{2}$ of the residuals between the observed and the synthetized light curve, but such an approach is unsatisfactory because the solution is unstable, i.e., small changes in the input data produce large changes in the spot map. This is due to the role played by the noise in the $\chi^{2}$ minimization, which implies that most of the structures appearing in the spot map actually come from the modelling of the light fluctuations due to intrinsic variability or measurement errors.
It is possible to overcome the uniqueness and stability problems by introducing a regularizing function into the solution process. This corresponds to the a priori assumption of some specific statistical property for the spot map, which allows us to select one stable map among the potentially infinite number of maps that can fit a given light curve. The two mostly used a priori assumptions applied to the star map problem are the Maximum Entropy (hereinafter ME; Gull \& Skilling 1984; Vogt et al. 1987) and the Tikhonov regularizations (hereinafter $\mathrm{T}$; Piskunov et al. 1990). They are particularly suited when the spot map consists of an array which specifies the spot covering factor in each surface element (or pixel) of the model star. The covering factor $f$ gives the specific intensity $I$ of each surface element according to the definition: $I=(1-f) I_{\mathrm{u}}+f I_{\mathrm{s}}$, where $I_{\mathrm{u}}$ is the specific intensity of the unspotted photosphere of the surface element, $I_{\mathrm{s}}$ that of the spotted photosphere, and $0<f<1$.

A detailed description of our modelling approach to the problem of light curve analysis, including the application of the ME and T regularizations, was presented by Lanza et al. (1998a) and we refer the reader to that paper for detailed information. Here we only recall that the ME spot map is computed by a constrained minimization of the functional:

$Q_{\mathrm{ME}}=\chi^{2}-\lambda_{\mathrm{ME}}\left(S_{1}+S_{2}\right)$

whereas the Tikhonov map comes from the constrained minimization of:

$Q_{\mathrm{T}}=\chi^{2}+\lambda_{\mathrm{T}}\left(T_{1}+T_{2}\right)$,

where $\chi^{2}$ is the normalized sum of the squared residuals between the observed and the synthetized light curves, $S_{k}$ is the entropy of the spot map of the $k$ th $\operatorname{star}(k=1,2)$, $T_{k}$ is the Tikhonov functional of the spot map of the $k$ th star and $\lambda_{\mathrm{ME}}$ and $\lambda_{\mathrm{T}}$ are the Lagrange multipliers for the Maximum Entropy and the Tikhonov regularizations, respectively. The explicit expressions for $\chi^{2}, S_{k}$ and $T_{k}$ and the procedure of evaluation of the Lagrange multipliers are described by Lanza et al. (1998a).

The synthetized light curve for a given distribution of the covering factor is computed using the computer code described by Lanza et al. (1998a), which has been improved by adopting Roche geometry to describe the surfaces of the components (which is well suited for SZ Psc, where the larger component has a radius of $82 \%$ of its Roche lobe) and Kurucz's (2000) atmospheric models to compute surface fluxes. The surface fluxes for a given gravity and effective temperature are computed adopting a simple linear limb darkening law to account for the angular dependence of the specific intensity. Such an approximation allows us to work with a constant projection matrix in the light curve inversion problem and to evaluate analytically the gradients of the objective functionals in Eqs. (1) and (2), thus reducing significantly the CPU time that is required to compute a solution (cf. Lanza et al. 1998a for details). The systematic errors introduced 
Table 1. $V$-band light curves of SZ Psc.

\begin{tabular}{|c|c|c|c|c|c|c|c|}
\hline $\begin{array}{l}\text { Light } \\
\text { curve }\end{array}$ & $\begin{array}{c}\text { Mid Epoch } \\
(\mathrm{JD}-2400000)\end{array}$ & $\begin{array}{l}\Delta T \\
(\mathrm{~d})\end{array}$ & $M$ & $\begin{array}{c}D_{\mathrm{T}} \\
(\mathrm{cm})\end{array}$ & $P$ & $\begin{array}{c}\text { Min. Epoch } \\
\text { HJD-2400000 }\end{array}$ & Reference \\
\hline 1957 & 36082.2 & 77 & 59 & 48 & $S$ & $36114.5740+3.96534286 \times E$ & Jakate et al. (1976) \\
\hline 1962 & 37953.2 & 95 & 18 & 48 & $S$ & $36114.5740+3.96534286 \times E$ & Jakate et al. (1976) \\
\hline 1974 & 42358.6 & 55 & 121 & 61 & $S$ & $36114.5740+3.96534286 \times E$ & $\begin{array}{l}\text { Jakate et al. (1976), } \\
\text { Bakos \& Tremko (1987) }\end{array}$ \\
\hline 1975 & 42671.0 & 175 & 33 & & $S$ & $36114.5740+3.96534286 \times E$ & Bakos \& Tremko (1987) \\
\hline 1976 & 43078.2 & 101 & 64 & 107 & $I, S$ & $36114.5740+3.96534286 \times E$ & Eaton (1977), Bakos \& Tremko (1987) \\
\hline 1977 & 43462.0 & 109 & 116 & $46-101$ & $I, S$ & $43823.6740+3.96582000 \times E$ & Eaton et al. (1982), Okazaki (1979) \\
\hline 1978 & 43809.3 & 116 & 243 & $38-152$ & $S$ & $43823.6740+3.96582000 \times E$ & Eaton et al. (1982) \\
\hline 1979 & 44142.6 & 140 & 87 & $40-48$ & $S$ & $43894.8850+3.96525000 \times E$ & $\begin{array}{l}\text { Tumer et al. (1980), } \\
\text { Percy \& Welch (1982) }\end{array}$ \\
\hline 1980 & 44566.0 & 75 & 72 & 40 & $I, S$ & $43823.6740+3.96582000 \times E$ & $\begin{array}{l}\text { Bakos \& Tremko (1987), Eaton (1980), } \\
\text { Percy \& Welch (1982) }\end{array}$ \\
\hline 1981 & 44781.9 & 100 & 193 & 48 & $S$ & $44827.0047+3.96578890 \times E$ & Tunca (1984) \\
\hline 1984 & 46006.4 & 133 & 25 & 40 & $I, S$ & $44827.0047+3.96578890 \times E$ & Bakos \& Tremko (1987), Percy (1984) \\
\hline 1986 & 46679.5 & 35 & 21 & $40-100$ & $S$ & $42309.1033+3.96540400 \times E$ & Thompson (1987) \\
\hline 1991 & 48947.1 & 48 & 40 & 75 & $S$ & $44827.0047+3.96578890 \times E$ & Doyle et al. (1994a) \\
\hline 1993 & 49282.5 & 123 & 19 & 80 & $S$ & $44827.0047+3.96578890 \times E$ & present paper \\
\hline 1994 & 49622.5 & 164 & 61 & 80 & $S$ & $44827.0047+3.96578890 \times E$ & present paper \\
\hline 1995 & 49965.5 & 138 & 22 & 80 & $S$ & $44827.0047+3.96578890 \times E$ & present paper \\
\hline 1996 & 50274.5 & 145 & 17 & 80 & $S$ & $44827.0047+3.96578890 \times E$ & present paper \\
\hline 1997 & 50758.5 & 103 & 13 & 80 & $S$ & $44827.0047+3.96578890 \times E$ & present paper \\
\hline $1998 \mathrm{a}$ & 51050.5 & 44 & 24 & 80 & $S$ & $44827.0047+3.96578890 \times E$ & present paper \\
\hline $1998 b$ & 51141.5 & 66 & 22 & 80 & $S$ & $44827.0047+3.96578890 \times E$ & present paper \\
\hline
\end{tabular}

by the linear limb darkening approximation have been reduced by computing its coefficients by means of the least square procedure proposed by Diaz-Cordoves et al. (1995) for Kurucz's models. Small errors in the atmospheric modelling give rise only to second-order effects to the purposes of our analysis of stellar activity, because it is based on the variation of the properties of the spot pattern from season to season. Absolute properties of the spots can not be extracted from single-band data because systematic errors arise from the unknown unspotted light level and the assumption of single-temperature spots.

\section{Model parameters}

All solutions were computed using squared map elements of side $s=18^{\circ}$ for both components. The masses and the radii of the components were taken from Popper (1988). The latest spectroscopic orbit derived by Marino (1999) confirmed the values of the stellar masses within a few per cent.

Roche lobe geometry was implemented according to Kopal (1989) with the Roche potential at the surface of each star computed from the mass ratio and the star's fractionary radius. The fractionary radii were slightly adjusted to improve the fits. Since the inclination of the orbital plane is $76^{\circ}$, the occulted strip on the visible portion of the disk ranges from latitudes $-76^{\circ}$ to $78.5^{\circ}$ for the $\mathrm{F} 8 \mathrm{~V}$ component and from $-76^{\circ}$ to $12^{\circ}$ for the $\mathrm{K} 1 \mathrm{IV}$ component, respectively.
The effective temperature of the unspotted photosphere of each star was derived from its spectral type and luminosity class according to Gray (1992). Surface gravity was computed according to the mass and radius after Popper (1988). Being the available infrared photometry quite sparse (e.g., Jakate et al. 1976; Eaton 1980; Antonopoulou \& Williams 1984; Thompson 1987), we assumed typical values for the spot effective temperatures, as derived by the analysis of other RS CVn binaries (cf., e.g., Eaton 1992). Spot temperatures were kept constant. This assumption would not produce significant errors into the geometry of the spot pattern, if the brightness of the spotted photosphere did not vary significantly from year to year. Solar observations support this assumption for the $V$-band, but brightness changes along the activity cycle might indeed occur in the infrared (Maltby 1992).

The latest photospheric models by Kurucz (2000) were used to evaluate the ratio of the specific intensities of the spotted and unspotted photospheres at the disk center $\left(C_{\mathrm{S}}\right)$ and the linear limb-darkening coefficients in the $V$-band $\left(u_{V}\right)$. Gravity darkening and reflection effect coefficients were introduced according to Lanza et al. (1998a).

All model parameters we adopted to compute the light curve fits are listed in Table 2, together with the corresponding references.

In order to fit all the light curves with constant values of the stellar fractional luminosities, at the outset we re-determined the luminosity ratio in the $V$-band, assuming it as an additional free parameter in the fitting procedure, as described by Rodonò et al. (1995) and 
Table 2. Geometrical and physical parameters adopted for modelling the SZ Psc light curves.

Orbital Elements of SZ Psc

\begin{tabular}{lcc}
\hline Element & & Ref. $\left({ }^{a}\right)$ \\
\hline Semi-major axis & 1.00 & \\
Eccentricity & 0.00 & 1 \\
Inclination (deg) & 76.0 & 1 \\
Time of conjunction & mid primary eclipse & \\
Period (day) & $\sim 3.966$ & 2 \\
\hline
\end{tabular}

Stellar and Model Parameters

\begin{tabular}{lccc}
\hline Stellar Parameter & F8 V & K1 IV & Ref. $\left({ }^{a}\right)$ \\
\hline Fractional radius & 0.0968 & 0.3293 & 1,4 \\
Mass $\left(M_{\odot}=1\right)$ & 1.28 & 1.62 & 1,3 \\
$V$-band fractional luminosity & 0.259 & 0.741 & 4 \\
Bolometric correction (mag) & -0.04 & -0.30 & 5 \\
Effective temperature $(\mathrm{K})$ & 6090 & 4910 & 6 \\
Surface gravity $\log g\left(\mathrm{~cm} \mathrm{~s}^{-2}\right)$ & 4.20 & 3.23 & 1 \\
Starspot temperature $(\mathrm{K})$ & 4900 & 3750 & 4,7 \\
Gravity darkening & 0.25 & 0.25 & 8 \\
$u_{V}$ (unspotted) & 0.683 & 0.778 & 9,10 \\
$u_{V}$ (spotted) & 0.778 & 0.824 & 9,10 \\
$C_{\mathrm{S}}$ & 0.348 & 0.132 & 9 \\
Bolometric albedo & 1.00 & 0.30 & 8 \\
Albedo in the $V$ pass band & 1.01 & 0.28 & 4
\end{tabular}

$\left(^{a}\right)$ References: [1]: Popper (1988); [2]: Hall \& Kreiner (1980);

[3]: Doyle et al. (1994a); [4]: Present paper; [5]: Allen (1973);

[6]: Gray (1992); [7]: Eaton (1992); [8]: Eaton et al. (1993);

[9]: Kurucz (2000); [10]: Diaz-Cordoves et al. (1995).

Lanza et al. (1998a). By minimizing the sum of the $\chi^{2}$ 's of the best covered light curves in the sequence, with $\lambda_{\mathrm{ME}}=0.5$ fixed, we derived the best value for the luminosity ratio in the $V$-band:

$\frac{L_{\mathrm{F} 8 \mathrm{~V}}}{L_{\mathrm{K} 1 \mathrm{IV}}}=0.350 \pm 0.035$.

The quoted uncertainty is an estimate of the range containing the true value of the luminosity ratio with $99 \%$ confidence level, when systematic errors are neglected (cf. Lampton et al. 1976; Rodonò et al. 2001). The above luminosity ratio led to the fractional luminosities reported in Table 2.

\section{Results}

\subsection{Light curve fitting and spot maps}

The light curve fits we obtained with the model parameters listed in Table 2 and the ME criterion are shown in Figs. 2 and 3, whereas those obtained with the same parameters and the T criterion are shown in Figs. 4 and 5, respectively. The corresponding values of the Lagrange multipliers and the $\chi^{2}$ 's are listed in Table 3. The $\chi^{2}$ 's were computed according to Eq. (3) in Lanza et al. (1998a), assuming a standard deviation $\sigma_{j}=3 \times 10^{-2} F_{0}$ for all the measurements, where $F_{0}$ is the unspotted flux of the system at quadrature. The fits are usually satisfactory and some small systematic deviations, especially during primary minima, can be accounted for by intrinsic light fluctuations of the system (see Sect. 2).

The derived distributions of the covering factor on the components are displayed using IDL and plotted on Mercator maps. Specifically, the original maps, having squared pixels with a side of $18^{\circ}$, were smoothed for presentation purposes to maps with a pixel side 5 times smaller using a bilinear interpolation (function REBIN of IDL) and then displayed as images (Figs. 6-9). On the maps of the primary the longitude is measured from the substellar point in the direction opposite to the orbital motion, whereas on the secondary it is measured from the antipodes of the substellar point, again in the direction opposite to the orbital motion. Thus on all the maps the phase at which a given spot element crosses the central meridian of the stellar disk is equal to its longitude.

The ME solutions show that polar spots are not required to fit the wide-band light curves, even during eclipses when the resolution is increased due to the occultation of one component by the other. However, it should be noted that the presence of polar spots can not be ruled out on the basis of photometric data alone, because eclipse mapping is little sensitive to the spot pattern in the polar regions due to foreshortening effects.

The spot pattern appears to be concentrated in the northern hemispheres of both components and extends up to $\sim 75^{\circ}$ in latitude. It is important to note that the rotation axes of the components are assumed to be perpendicular to the orbital plane, so that the north poles of both stars are always in view. Since the ME regularization tends to reduce the spot area as much as possible, the northern hemispheres of the components are preferred for spot location because of the smaller foreshortening angles.

Several small structures are present on the hemispheres occulted during eclipses, but they are the result of image artifacts due to the ME regularization, the so-called super-resolution effect (Narayan \& Nityananda 1986). The actual resolution, deduced from the analysis of several simulated light curves with a pseudo-Gaussian noise of $\sigma=0.02 \mathrm{mag}$ and $M=40$ normal points, is $\sim 20^{\circ}-30^{\circ}$ on the strips occulted during eclipses and $\sim 40^{\circ}-60^{\circ}$ outside of them. Light curves with the same $\sigma$ and a larger $M$ have a somewhat higher resolution, but structures smaller than $30^{\circ}-40^{\circ}$ should in any case be regarded with caution because they might be artifacts, even within the eclipsed strips.

An alternative description of the spot pattern is provided by the maps regularized with the $\mathrm{T}$ criterion. Those maps are the smoothest that fit the light curves. They show that several details in the ME maps are not necessarily required by the data.

The $\mathrm{T}$ maps of the $\mathrm{F} 8 \mathrm{~V}$ primary are quite different from the ME maps, although both show a concentration of spots around the central meridian of the hemisphere occulted at primary eclipse. Their difference can be attributed to the lower luminosity of the primary that makes the photometric contribution of its surface 
inhomogeneities difficult to detect for contrast reasons, except when the star's disk is occulted and the light curve profile is more sensitive to their effect. Therefore, only the spotted structures on the F8 V star occulted at the ingress and egress of the primary eclipses can be regarded with some confidence. Structures at longitudes corresponding to phases out of primary eclipses should be considered with caution, particularly during years with gaps in the phase coverage.

The prominent spot features on the photosphere of the F8 V primary, as derived from the best covered primary minima (i.e., 1978, 1979, 1981), have diameters between $40^{\circ}$ and $60^{\circ}$ both on the ME and T maps, i.e., comparable to the geometrical resolution of the eclipse mapping, and suggest that even smaller, solar-sized spots might be present on the star. They may not be confined to the occulted hemisphere, but are likely to be present at other longitudes on the star, although their photometric effect is too little to be unambiguously detected.

The features on the ME and the T maps of the K1 IV secondary are remarkably similar, even if several small details appearing on the ME maps are not found on the $\mathrm{T}$ maps. In particular, the secondary eclipse modulation can be reproduced, in most cases, by only one large and smooth spot centered around longitudes $150^{\circ}-180^{\circ}$. On the maps derived from good covered secondary minima in 1976, 1978 and 1979, its shape and extension varies in time and it shows a circular and compact core, with a diameter of $\sim 40^{\circ}-50^{\circ}$. On the map of the 1981 light curve, the compact core has disappeared and the spotted area extends more or less uniformly between longitudes $\sim 120^{\circ}-200^{\circ}$. Other active regions seem to be present around longitudes $\sim 50^{\circ}$ and $\sim 270^{\circ}$ although they are not as stable as the active region around longitude $\sim 180^{\circ}$ and show remarkable changes of location and extension from one year to another.

A drawback of the $\mathrm{T}$ regularization is the presence of a high latitude spot pattern, which is not actually needed to fit the data, as shown by the ME fits. Photometric data give very poor constraints on the high latitude features which are dominated by the regularization. This produces an extension of some features towards the poles and a uniformly grey area at high latitudes, smoothing out the variation of the covering factor over the star surface and increasing the total spotted area with respect to the ME maps.

The distributions of the spotted area vs. longitude are shown in Figs. 10 and 11 for the F8 V primary component and in Figs. 12 and 13 for the K1 IV secondary component, respectively. The plotted distributions are those derived from the actual spot covering factor, binned into $18^{\circ}$ longitude intervals, without any smoothing with the IDL REBIN function. The ME distributions give the lowest area per longitude bin actually required by the data, for the assumed system parameters and unspotted magnitude. The relative uncertainties are within the dimensions of the plotting symbols (cf. Lanza et al. 1998a for more details on the accuracy of the derived spot areas).
The maps obtained with the $\mathrm{T}$ regularization usually show a significantly larger spotted area per longitude bin than those obtained with the ME criterion, due to the presence of the high latitude "grey caps".

Each distribution of spotted area vs. longitude can be represented as the sum of a uniform term $a_{\mathrm{U}}$, which is defined as the minimum spotted area at all longitudes, and a term $a_{\mathrm{D}}(l)$ which varies vs. longitude $l$. The latter term often shows more than one relative maximum and minimum, thus suggesting the presence of several preferential longitudes.

Table 3. The Lagrangian multipliers and the $\chi^{2}$ of the light curve fits with ME and T regularizing criteria, respectively.

\begin{tabular}{ccccc}
\hline Light curve & $\lambda_{\mathrm{ME}}$ & $\chi_{\mathrm{ME}}^{2}$ & $\lambda_{\mathrm{T}}$ & $\chi_{\mathrm{T}}^{2}$ \\
\hline 1957 & 0.5 & 0.572 & 40.0 & 0.886 \\
1962 & 0.5 & 1.495 & 40.0 & 1.963 \\
1974 & 0.25 & 0.688 & 40.0 & 0.833 \\
1975 & 1.0 & 0.940 & 90.0 & 0.929 \\
1976 & 0.25 & 0.503 & 40.0 & 0.826 \\
1977 & 1.5 & 1.687 & 40.0 & 1.554 \\
1978 & 1.0 & 2.093 & 40.0 & 2.163 \\
1979 & 1.0 & 5.743 & 40.0 & 6.073 \\
1980 & 1.5 & 0.709 & 40.0 & 0.721 \\
1981 & 0.25 & 0.710 & 40.0 & 0.800 \\
1984 & 2.0 & 6.491 & 90.0 & 6.309 \\
1986 & 0.5 & 0.362 & 90.0 & 0.327 \\
1991 & 2.0 & 1.236 & 90.0 & 1.064 \\
1993 & 2.0 & 0.264 & 90.0 & 0.191 \\
1994 & 0.5 & 0.542 & 90.0 & 0.697 \\
1995 & 1.5 & 1.086 & 90.0 & 1.499 \\
1996 & 1.5 & 0.276 & 90.0 & 0.474 \\
1997 & 1.5 & 1.851 & 40.0 & 2.312 \\
$1998 \mathrm{a}$ & 1.0 & 1.296 & 90.0 & 1.372 \\
$1998 \mathrm{~b}$ & 0.5 & 0.712 & 40.0 & 0.868 \\
\hline
\end{tabular}

\subsection{Overall evolution of the spot pattern}

The total area covered by spots, $A=A_{\mathrm{U}}+A_{\mathrm{D}}$, and the total areas of the uniform and longitude-dependent components, $A_{\mathrm{U}}=\int_{0}^{2 \pi} a_{\mathrm{U}} \mathrm{d} l$ and $A_{\mathrm{D}}=\int_{0}^{2 \pi} a_{\mathrm{D}} \mathrm{d} l$, are plotted vs. time in Figs. 14a-c for the F8 V primary component and in Figs. 15a-c for the K1 IV secondary component, respectively. The relative uncertainties of the values of $A$ related to the stability of the solutions are between $\sim 25 \%-50 \%$ for the primary component and $\sim 5 \%-10 \%$ for the more luminous secondary. An additional source of uncertainty is the non-standard photometric system of some light curves, but the advantage of analysing data in different systems is that the errors tend to become stochastic.

The total spotted areas on the F8 V star, as derived from the ME and the T maps, are markedly different. This indicates that the information content of the light curves on the spot pattern of the primary component is very low. The ME maps, which tend to minimize the spot coverage 


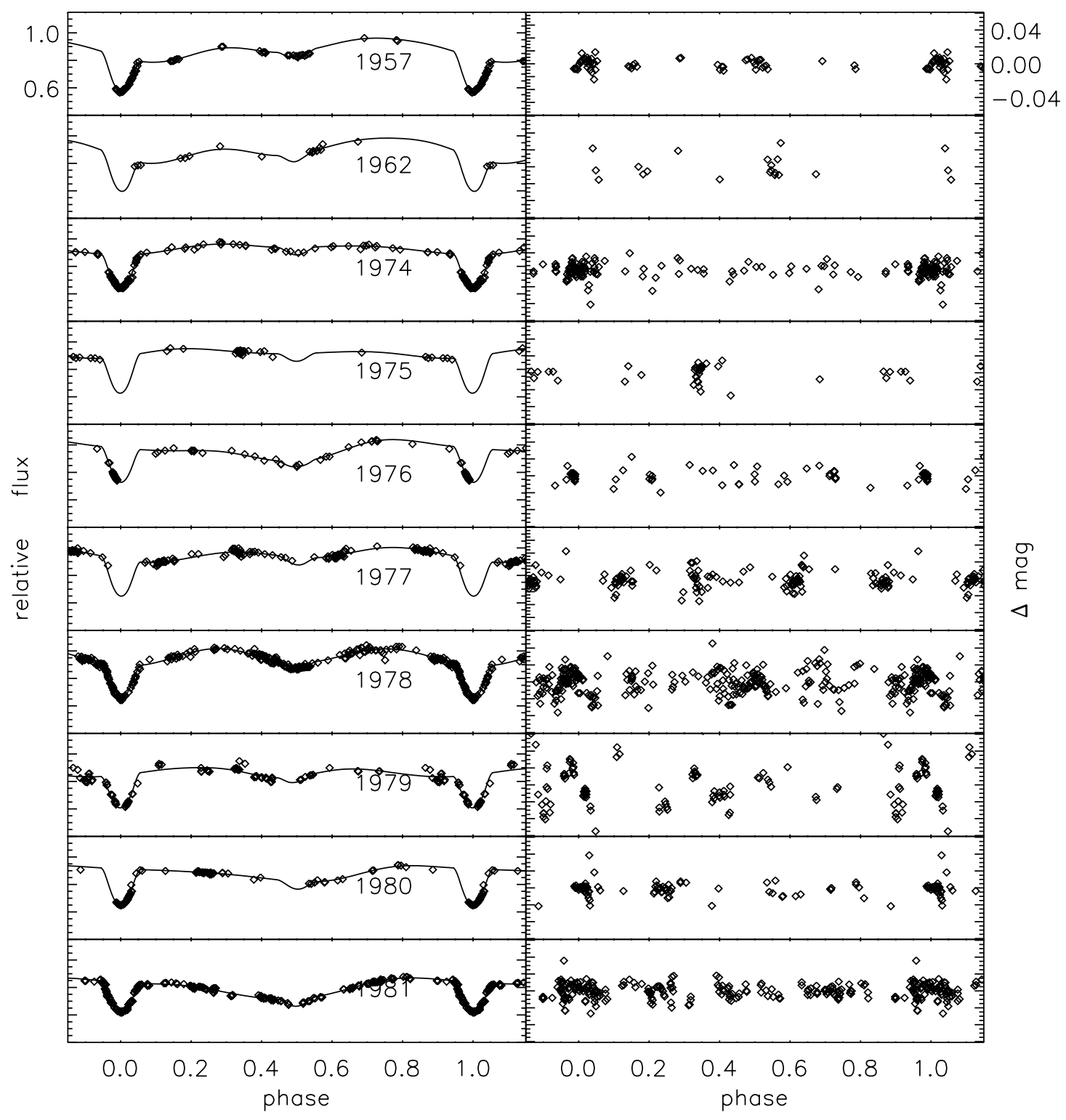

Fig. 2. The $V$-band light curve fits with the Maximum Entropy regularization criterion from 1957 to 1981 (left panels) and the corresponding residuals (right panels). Zero phase corresponds to mid primary eclipse. Note that the right panel scale is about six times more expanded than the left panel scale.

fraction, indicate that at most $\sim 5 \%$ of the stellar photosphere should be covered by spots in order to fit the light curves, even in the years of the highest activity level. No definite conclusion can be drawn on the spot evolution from the information provided by our light curves, except some indication for the presence of a fairly stable active region around the substellar point facing the other component. Such a spotted area, however, showed significant changes from the $1978-1979$ to the 1981 seasons, being more extended in the latter season. However, a study of its time evolution is prevented by the small number of light curves with a complete coverage of the primary minimum.

The total spotted area of the K1 IV secondary ranges from $\sim 8 \%$ to $\sim 28 \%$ both in the $\mathrm{ME}$ and $\mathrm{T}$ maps. Both regularization methods give almost the same values for the total spotted area because the information content of the light curves is higher for the more luminous secondary star. A periodogram analysis with the Scargle method (Scargle 1982; Horne \& Baliunas 1986) suggests that the T area may undergo cyclic changes with a period of $13.2 \mathrm{yr}$. However, the confidence level for such a detection is only $80 \%$ and the periodogram analysis of the ME data warns us that a periodicity with a period of $12.6 \mathrm{yr}$ may be an alias of a periodicity of $374 \mathrm{~d}$, probably depending on the schedule adopted for the observations. In any case, it seems that one cycle of $\sim 12-13$ yr occurred, as it is apparent from the modulation of the total spotted area observed between 1974 and 1986 (cf. Fig. 15a). The shape of the modulation is markedly non-sinusoidal and it does not appear to repeat regularly in the successive years. This cycle is more clearly apparent in the modulation of the spotted area unevenly distributed in longitude (cf. Fig. 15c). 


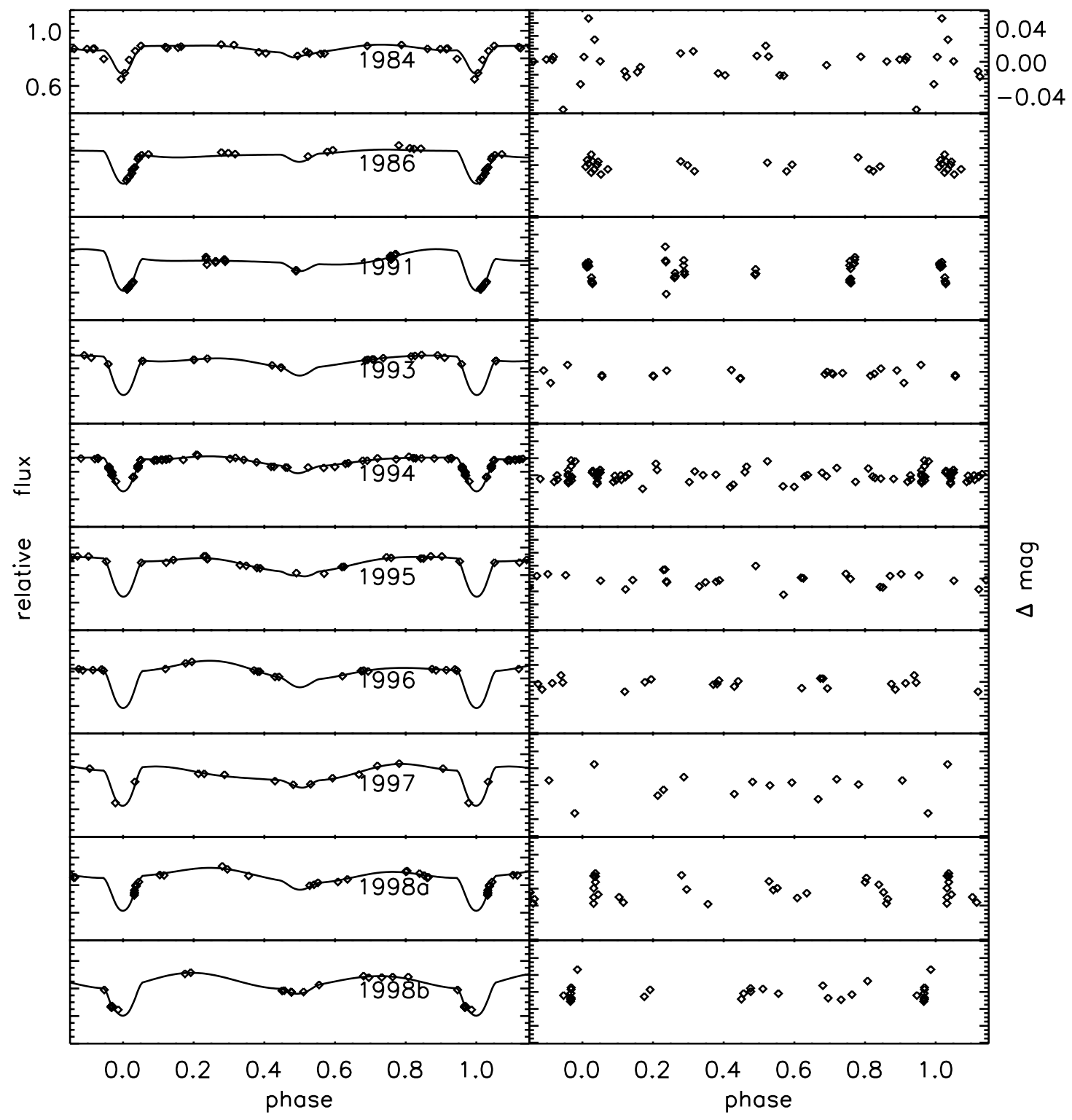

Fig. 3. As Fig. 2 for the light curves in the time interval 1984-1998.

The total spotted area on the K1IV component decreased from 1957 to 1962 and then remained approximately constant to $\sim 10 \%$ of the star's surface until 1978 . A rapid increase occurred between 1978 and 1979 and the spot area kept on rising up to $\sim 28 \%$ at activity maximum in 1981. After that maximum, the area showed oscillations around an average value of $\sim 18 \%$, without any clearly apparent periodicity, up to 1998 .

Three active longitudes are usually present on the secondary, but only one appears to be permanent around the substellar point facing the primary. Their evolution is readily apparent from the ME maps. The active longitude around $\sim 50^{\circ}-100^{\circ}$ is more compact than the two others at the substellar point and around $\sim 270^{\circ}-360^{\circ}$.

No steady migration of the spot pattern in longitude is apparent. There is some evidence for an episodic migration of the overall spot pattern in longitude, e.g., between 1984 and 1986, or 1997 and 1998a. However, it is really difficult to judge whether we were observing the overall motion of a more or less constant pattern or simply the effect of evolutionary changes within large and steady active regions.

\subsection{Orbital period variation}

The epochs of minima listed by Hall \& Kreiner (1980) were considered, together with some recently determined epochs that are listed in Table 4, to study the variation of the orbital period of SZ Psc. The epochs in the present study were estimated from the phase shifts that were required to obtain optimal fits for the 1994 and 1998b light curves. The APT observations seldom provide a complete coverage of the primary minima, as in the case of photometric observations dedicated to eclipse timing. Therefore, the accuracy of the determined epochs is not high, we estimated to be of the order of 0.01 in phase, i.e., 0.04 days. 


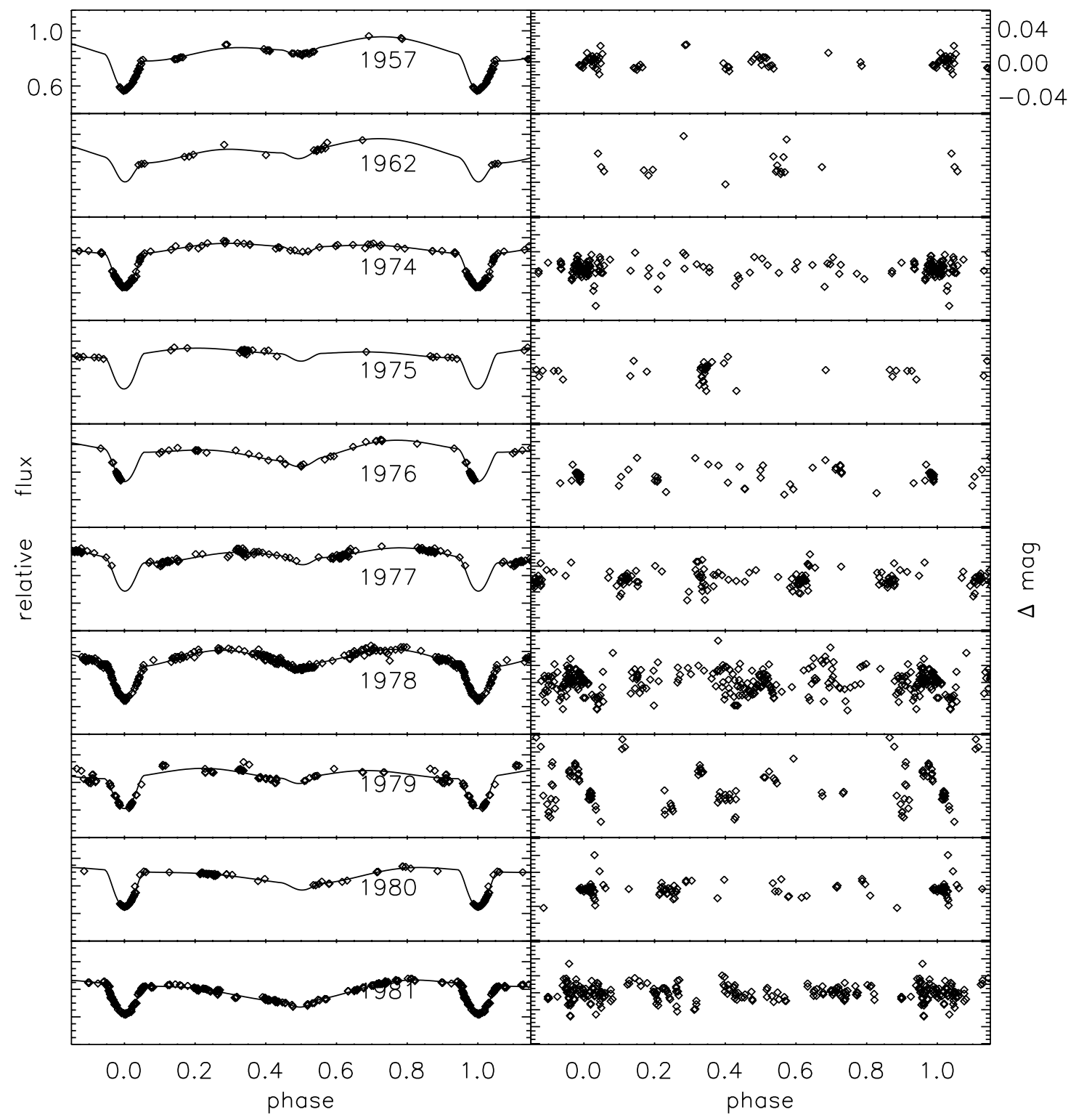

Fig. 4. The $V$-band light curve fits with the Tikhonov regularization from 1957 to 1981 (left panels) and the corresponding residuals (right panels). Zero phase corresponds to mid primary eclipse. Note that the right panel scale is about six times more expanded than the left panel scale.

The O-C's were computed with respect to the ephemeris given by Tunca (1984):

$$
\operatorname{Min} I=2444827.0047+3.9657889 \times E
$$

and were plotted versus time in Fig. 16 ( $E$ is the number of orbital cycles elapsed from the minimum in Eq. (4)). The O-C's obtained before HJD 2430000 were based on visual or photographic observations and their accuracy is rather poor (cf. Hall \& Kreiner 1980). The uncertainties in these minima epochs give rise to errors of about $0.05 \mathrm{~d}$ in the corresponding $\mathrm{O}-\mathrm{C}$ 's. We refer only to primary minima because secondary minima are too shallow to be useful for orbital period studies in the present case, in which light curves are significantly distorted by spot activity. Such distortions may affect also the primary minima, but the uncertainty of their epochs does not exceed $\sim 0.03 \mathrm{~d}$, as can be estimated from the standard deviation of the photoelectrically determined $\mathrm{O}-\mathrm{C}$ 's and the formula given by Hall \& Kreiner (1980).

It is important to note that the shape of the $\mathrm{O}-\mathrm{C}$ plot depends on the adopted ephemeris. Kalimeris et al. (1995) made a Fourier analysis of the O-C data up to 1995 and suggested a periodicity of $56 \mathrm{yr}$, that is comparable with the time extension of the data. Our new data seem to indicate an approximately semisinusoidal variation, which might be part of a complete cycle with a period of roughly $60 \mathrm{yr}$. Such a long-term periodicity, if confirmed, does not appear to be simply related with the possible $13 \mathrm{yr}$ activity cycle of the K1IV secondary. However, the longterm variation of the spot coverage between 1957 and 1984 


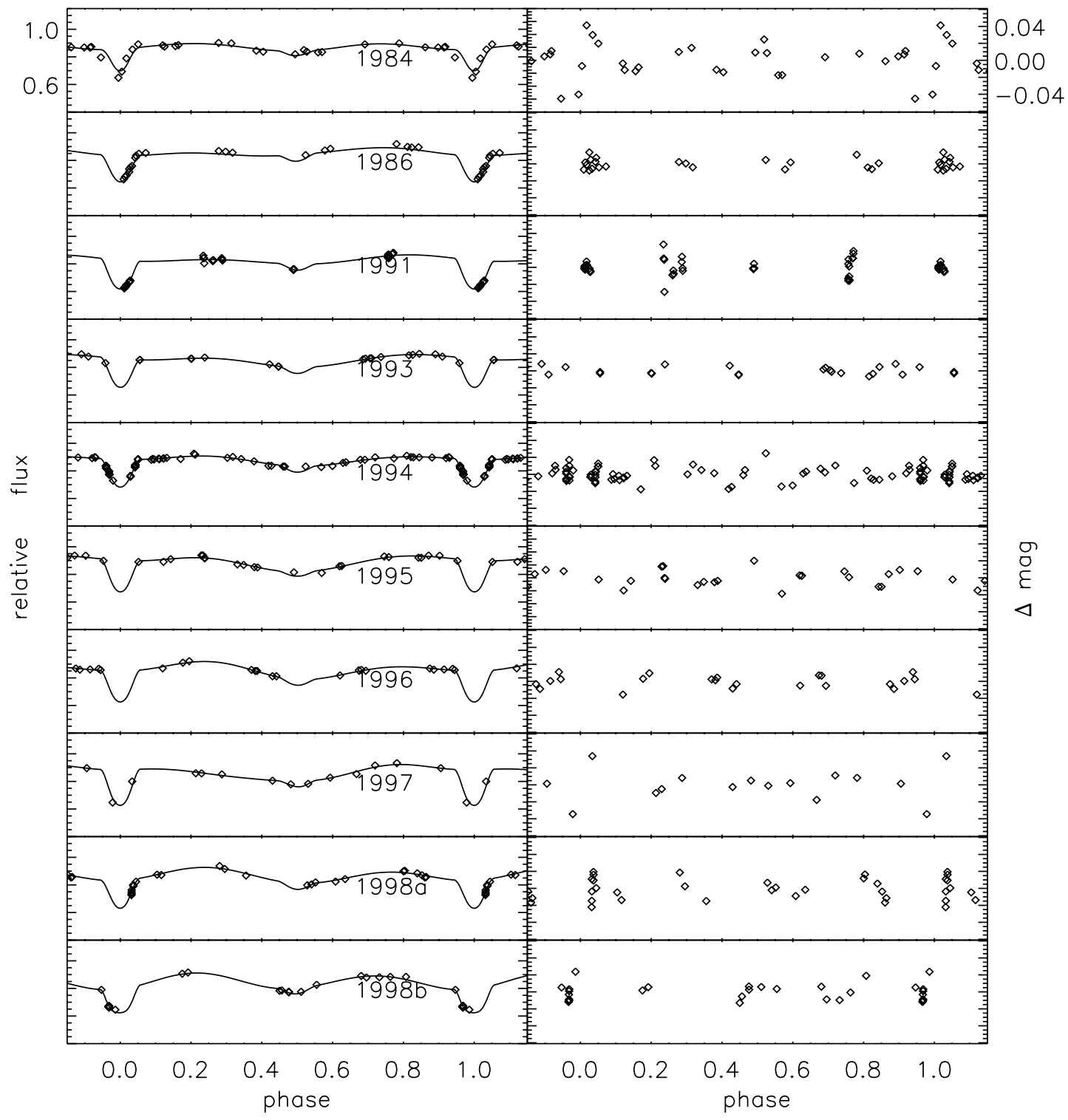

Fig. 5. As Fig. 4 for the lightcurves in the time interval 1984-1998.

might point towards a non-sinusoidal cycle with a period of $\approx 30 \mathrm{yr}$, approximately half of the possible orbital period modulation.

The spot activity on the secondary and the orbital period change are compared in Fig. 17, where the total fractional spot area (panel a) and $\mathrm{O}-\mathrm{C}$ 's (panel b) are plotted versus time. We see that the orbital period decrease between 1957 and 1980 was accompanied by a decrease of the spot area, although the lack of photometric data between 1962 and 1974 prevents us from drawing a definite conclusion. Since 1980, the orbital period has been approximately constant (almost constant $\mathrm{O}-\mathrm{C}$ values), and the spot area has been fluctuating around a roughly constant level.

\section{Discussion}

The luminosity ratio in the $V$ passband was determined through an optimization procedure using the light curves
Table 4. Recent epochs of primary minima of SZ Psc.

\begin{tabular}{ll}
\hline Epoch (HJD) & Reference \\
\hline 2443823.6740 & Eaton et al. (1982) \\
2444557.3156 & Bakos \& Tremko (1987) \\
2444827.0047 & Tunca (1984) \\
2449625.72 & present work \\
2451140.65 & present work \\
\hline
\end{tabular}

with the most complete phase coverage, as already done for RS CVn and AR Lac in previous papers (cf. Rodonò et al. 1995; Lanza et al. 1998a). The value we found, $L_{\mathrm{F} 8 \mathrm{~V}} / L_{\mathrm{K} 1 \mathrm{IV}}=0.350 \pm 0.035$, compares well with the previous estimate by Eaton et al. (1982), who found $L_{\mathrm{F} 8 \mathrm{~V}} / L_{\mathrm{K} 1 \mathrm{IV}}=0.34$. They used the Russell model to determine the binary parameters and applied it to the 1978 $V$ light curve, after removing the distortion produced by spots by means of a heuristic method. 


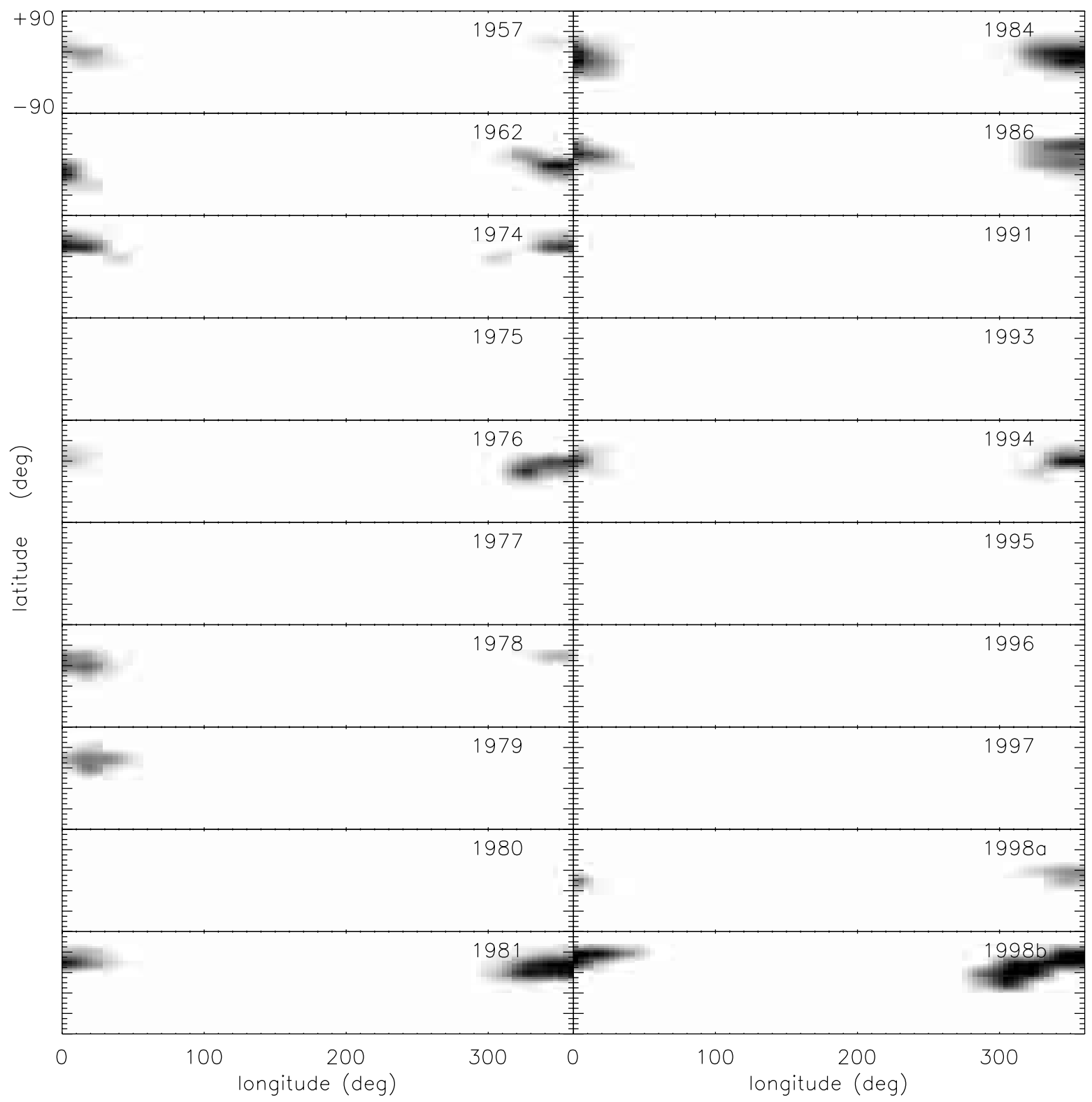

Fig. 6. The distribution of the spot covering factor on the F8 V primary from Maximum Entropy regularized fits of the $V$-band light curves from 1957 to 1998.

It is important to note that our approach is based on fitting all the light curves in our sequence by using the same unspotted luminosity ratio, i.e., that corresponding to the components without spots on their photospheres. Other authors have chosen to include the luminosity ratio among the varied parameters, allowing it to change from one light curve solution to another (e.g., Kang \& Wilson 1989). We prefer to assume a constant value because it is difficult to justify changes of the effective temperatures of the unspotted photospheres related to magnetic activity on timescales of a few decades (Spruit 1982; Spruit $\&$ Weiss 1986). It should be noted that the value of the unspotted luminosity ratio determined in the present analysis depends on the value adopted for the unspotted magnitude of the system, that in our case is the magnitude at the maximum brightness ever observed. Its dependence on other photometric parameters is much smaller and it was not investigated in the present study because we intend to focus on the analysis of the magnetic activity. More information can be found in a paper by Rodonò et al. (2001), where a method for the simultaneous determination of the luminosity ratio and the other photometric parameters (relative radii, orbital inclination, etc.) is presented. We plan to apply it also to SZ Psc in a forthcoming study to investigate the physical properties and the evolutionary status of the components of the system.

In the present analysis we allow spots to be present on both components. As a matter of fact, spectroscopic evidence for magnetic activity in the F8 V primary is very little (Huenemoerder \& Ramsey 1984; Doyle et al. 1994a; Marino 1999). However, it has a shallow outer convective envelope that may support dynamo action. Therefore, we 


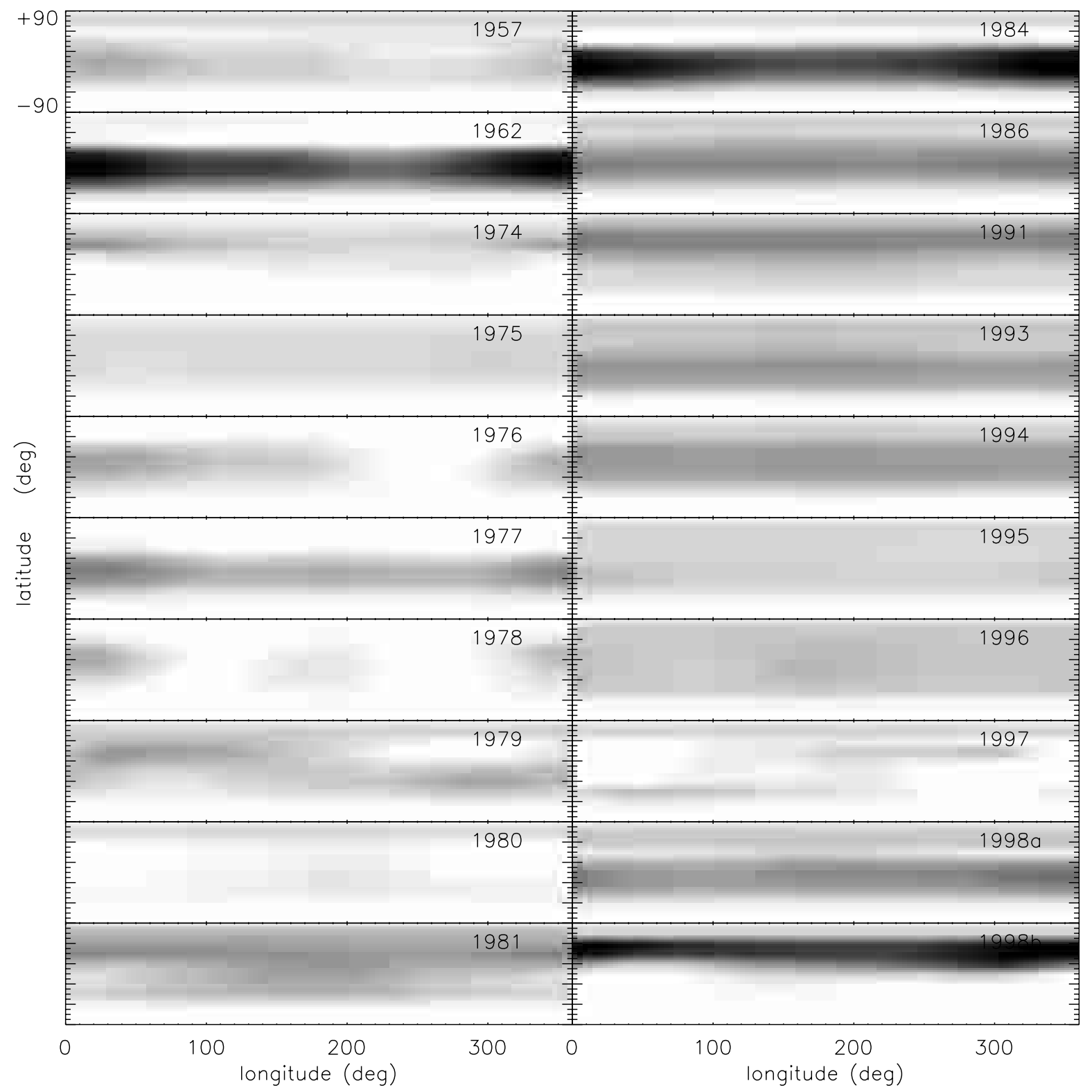

Fig. 7. The distribution of the spot covering factor on the F8 V primary from Tikhonov regularized fits of the $V$-band light curves from 1957 to 1998.

consider that spot activity may take place also on the primary component. The allowance for spots on the primary may improve the fit of the ingress and egress branchs of the primary eclipses, in particular allowing us to reproduce accurately the slope changes. The increase of resolution during primary eclipses allows us to perform an eclipse mapping of the primary and to provide some hints on the presence of spots around the substellar point. However, the available information is always very limited and no firm conclusion can be drawn on the spot activity of the primary. An unambiguous mapping of the photospheric inhomogeneities of the primary must await the application of techniques based on high-resolution spectroscopy such as Doppler imaging (cf. Vincent et al. 1993).

The information content of the light curves is adequate to characterize the main features of the spot activity of the more luminous K1 IV secondary. We applied simultaneously two different regularization techniques in order to point out which features are actually needed by the data and which are artifacts of the regularization itself. As already noted by Lanza et al. (1998a), the application of $\mathrm{ME}$ and $\mathrm{T}$ techniques to photometric data produces results that require a more careful interpretation than in the case of the Doppler imaging maps, which are based on high-resolution spectroscopic data with an intrinsically greater information content. Even in the case of ideal light curves with a perfect phase coverage and infinite signal-to-noise ratio, the regularization techniques introduce artifacts. In the case of the ME regularization they mainly consist of the so-called super-resolution, i.e., the presence of compact and highly contrasted features, which can not be actually resolved by means of 


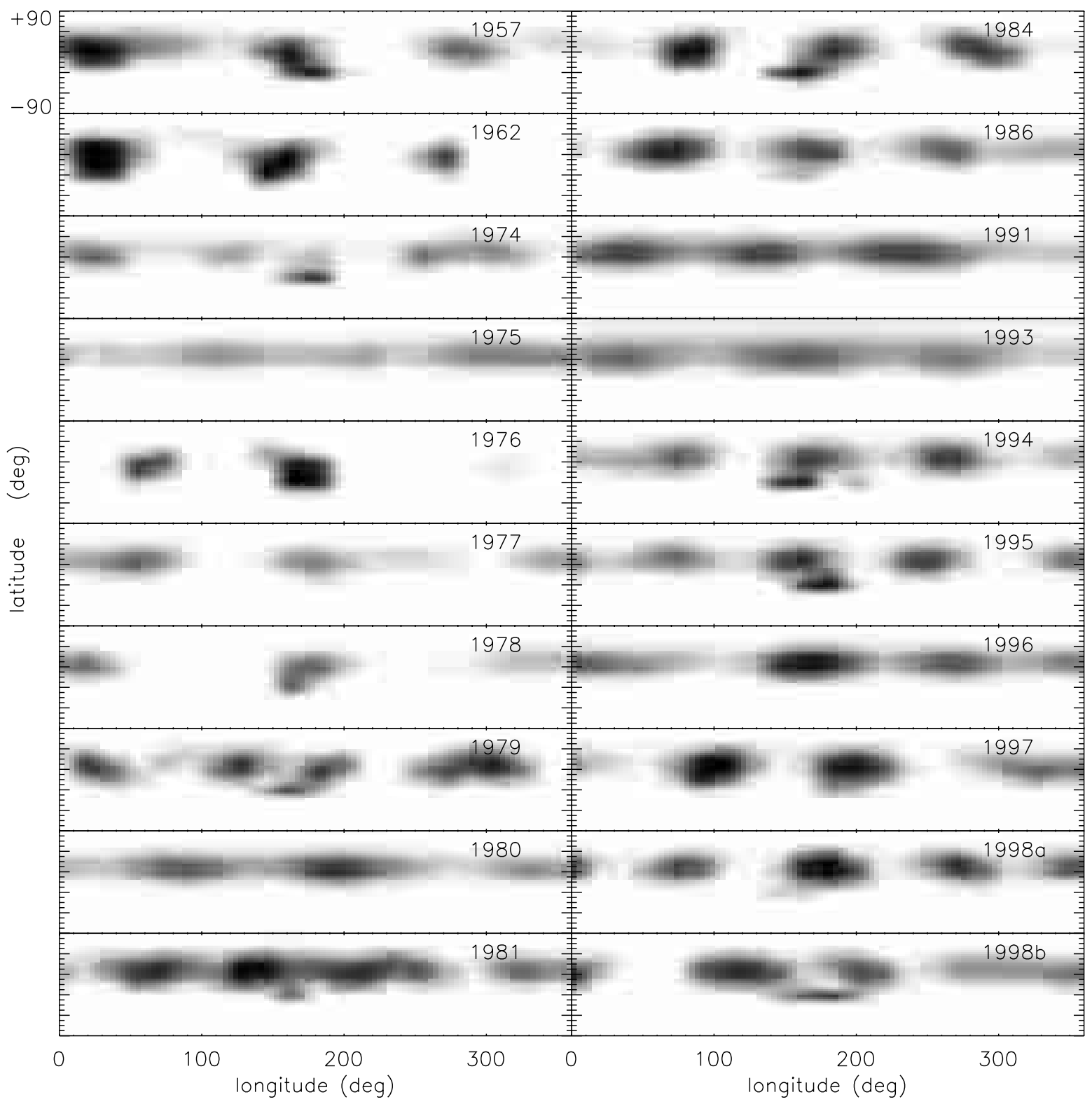

Fig. 8. The distribution of the spot covering factor on the K1 IV secondary from Maximum Entropy regularized fits of the $V$-band light curves from 1957 to 1998.

photometric techniques. On the contrary, the T regularization tends to produce uniformly spotted gray areas in those regions that do not contribute significantly to the light curve modulation, such as the polar regions in an eclipsing system that are strongly subject to foreshortening. A detailed description of the causes of such artifacts can be found in Lanza et al. (1998a). From these arguments, it is not possible to specify an ideal regularizing criterion for wide-band light curve analysis. In our opinion, the best approach is to regard regularized maps as an intermediate stage of the analysis and use them only to derive quantities which do not depend on the regularizing criterion itself, e.g., the spot longitudes, their changes in time and the variations of the total spotted area. Absolute values of the spotted areas are not reliable since they depend on the unspotted magnitude and the regularizing criterion adopted, but their time changes show a very small dependence on both of them. See, in particular, Fig. 15 concerning the secondary component, for which the information content of the data is sufficiently high.

The presence of active longitudes on the K1 IV secondary is an interesting phenomenon. Activity was persistently observed around the substellar point on the hemisphere facing the primary. The active region appears to be quite compact, also on the $\mathrm{T}$ maps, with a diameter of $\sim 40^{\circ}$, near the resolution limit of the eclipse mapping technique. It cannot be an artifact due to an incorrect treatment of the proximity effects because it displayed changes of shape and location. The other active longitudes are less well defined, especially on the T maps, and show marked changes from season to season. The orbital period of SZ Psc is close to an integer multiple of a day, making 


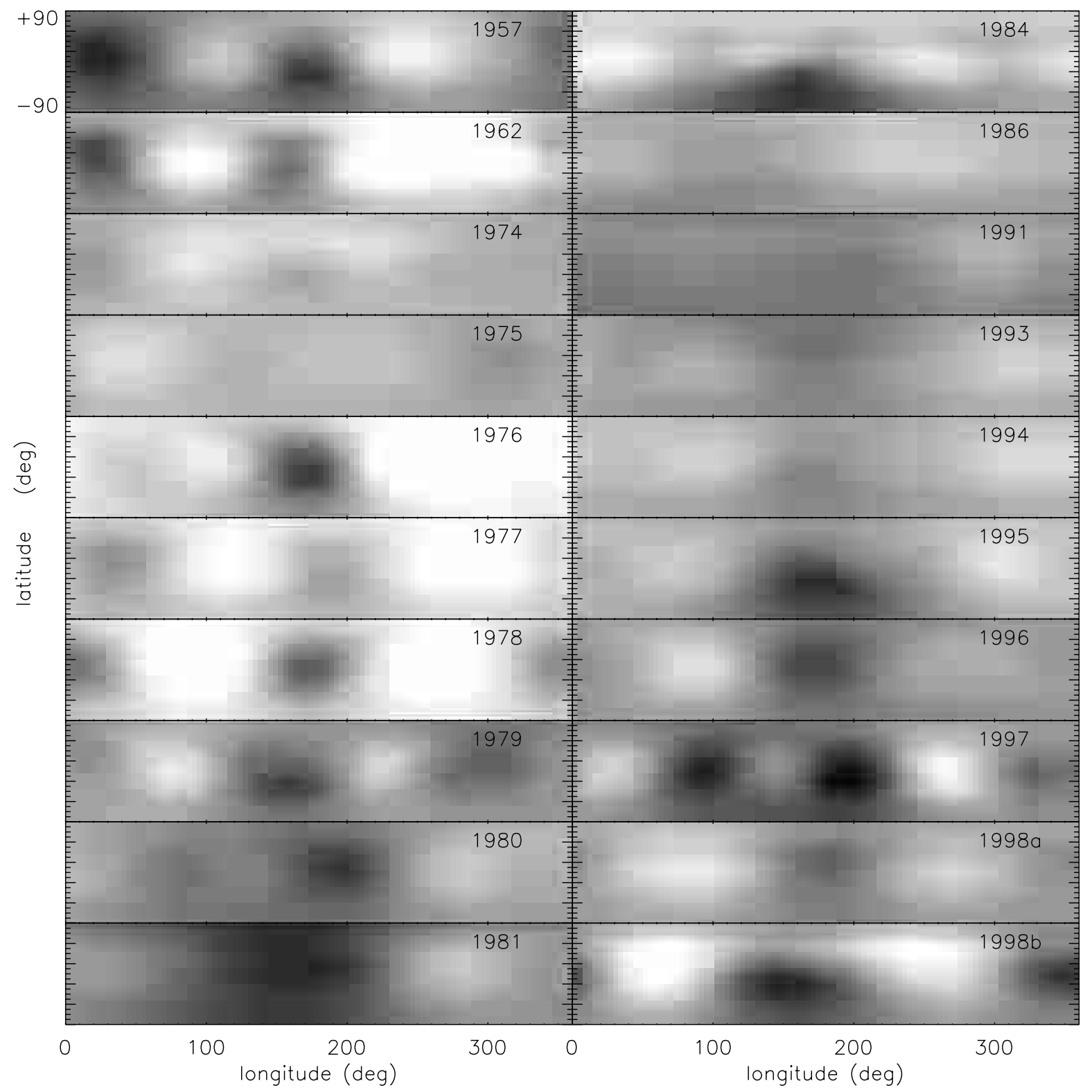

Fig. 9. The distribution of the spot covering factor on the K1 IV secondary from Tikhonov regularized fits of the $V$-band light curves from 1957 to 1998.

it difficult to achieve adequate phase coverage in a short time. Given the short-term fluctuations of the system magnitude, which probably corresponds to short-term changes of the spot pattern, it is difficult to derive unambiguous information on the unsteady active longitudes.

The esistence of active longitudes is a common phenomenon in RS CVn binaries (e.g., Henry et al. 1995; Oláh et al. 1997; Rodonò et al. 2000). The phenomenology observed on the secondary of SZ Psc compares well with that of the K0 IV secondary of AR Lac. Both stars show persistent active regions located around the substellar points on the hemispheres facing their respective companion stars. The other active longitudes are less well defined and show quite large changes of location and spotted area. No overall migration of the spot pattern in longitude has been detected, both for SZ Psc and AR Lac. Previous claims for a fast migration of the photometric wave (Percy \& Welch 1982; Blanco et al. 1983; Bakos \& Tremko 1987) may be explained by the attempts to fit only the out-of-eclipse portions of the light curves with simple sinusoidal waves, while the actual light curve changes are more complex and their distortions can be markedly non-sinusoidal.

The secondary component of SZ Psc shows clear evidence of activity also at the chromospheric and transition region levels (Huenemoerder \& Ramsey 1984; Popper 1988; Doyle et al. 1994a, 1994b; Marino 1999). Huenemoerder \& Ramsey (1984) found that the excess $\mathrm{H} \alpha$ emission of the K1 IV star had no clear phase dependence, although there was a puzzling flattening and broadening of the excess $\mathrm{H} \alpha$ profile near conjunctions.

In addition to flare activity, a compact plage was detected by Doyle et al. (1994a) in the Mg II h\&k lines, 


\begin{tabular}{|c|c|c|}
\hline 0.030 & & \\
\hline 0.000 & 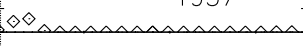 & 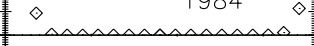 \\
\hline & 1962 & 1986 \\
\hline & & \\
\hline & 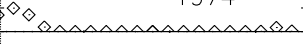 & 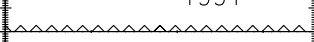 \\
\hline ¿ & 1975 & 1993 \\
\hline & 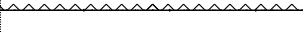 & $\Delta \Delta \Delta \Delta \Delta \Delta \Delta \Delta \Delta \Delta \Delta \Delta$ \\
\hline & 1976 & 1994 \\
\hline & 1977 & 1995 \\
\hline 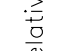 & 1978 & 1996 \\
\hline & 1979 & 1997 \\
\hline & & \\
\hline & 1980 & $1998 a$ \\
\hline & 1981 & $1998 \mathrm{~b} \diamond \diamond$ \\
\hline & $\begin{array}{l}100200 \quad 300 \\
\text { longitude (deg) }\end{array}$ & $\begin{array}{l}0 \quad 100 \quad 200300 \\
\text { longitude (deg) }\end{array}$ \\
\hline
\end{tabular}

Fig. 10. The distribution of the relative spot area vs. longitude obtained from the ME models for the F $8 \mathrm{~V}$ primary. The area unit is the area of the star photosphere (see text).

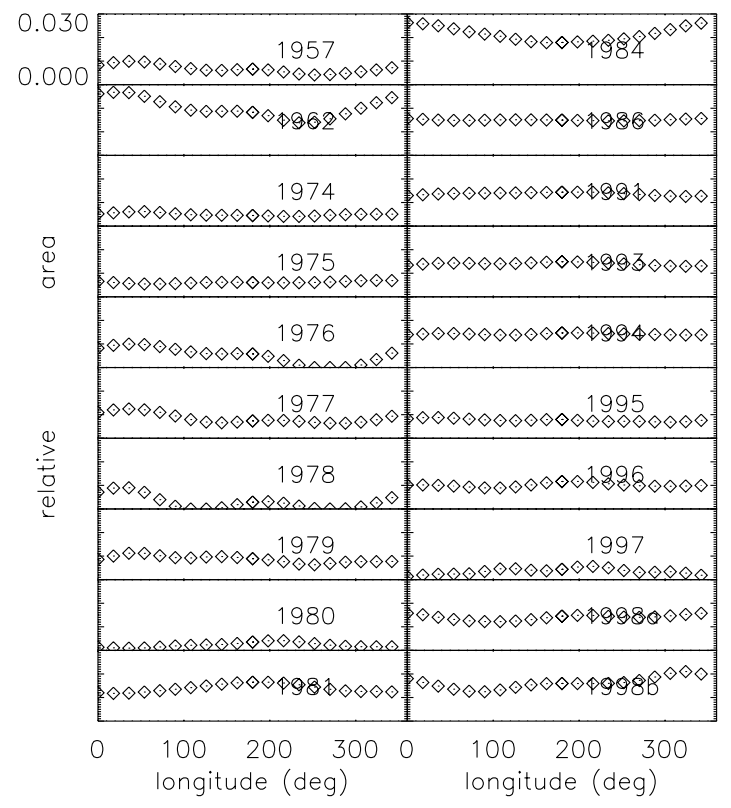

Fig. 11. The distribution of the relative spot area vs. longitude obtained from the $\mathrm{T}$ models for the $\mathrm{F} 8 \mathrm{~V}$ primary. The area unit is the area of the star photosphere (see text).

located on the hemisphere facing the primary component. Therefore, there is some evidence for chromospheric activity spatially associated with the active longitude around the substellar point, although the photometric observations used to derive the spot map are not strictly simultaneous with the UV spectroscopy. The O I $\lambda 1305$ and $\mathrm{C}$ II $\lambda 1135$ lines also showed a drop in flux at secondary minimum, but higher temperature lines, such as C IV $\lambda 1550$ and N v $\lambda 1240$, did not show phase-related flux changes. Such a behaviour is reminiscent of the active region

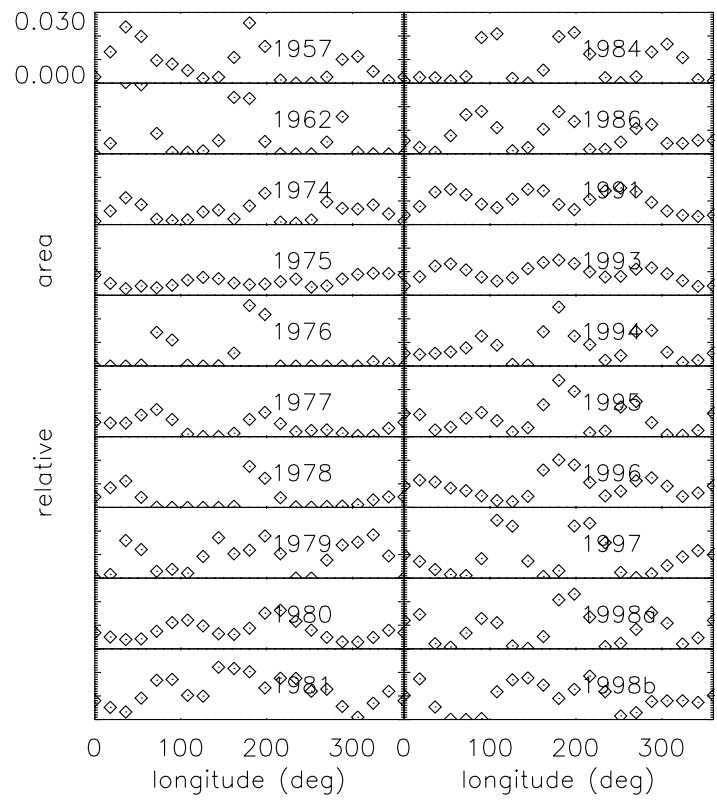

Fig. 12. The distribution of the relative spot area vs. longitude obtained from the ME models for the K1 IV secondary. The area unit is the area of the star photosphere (see text).

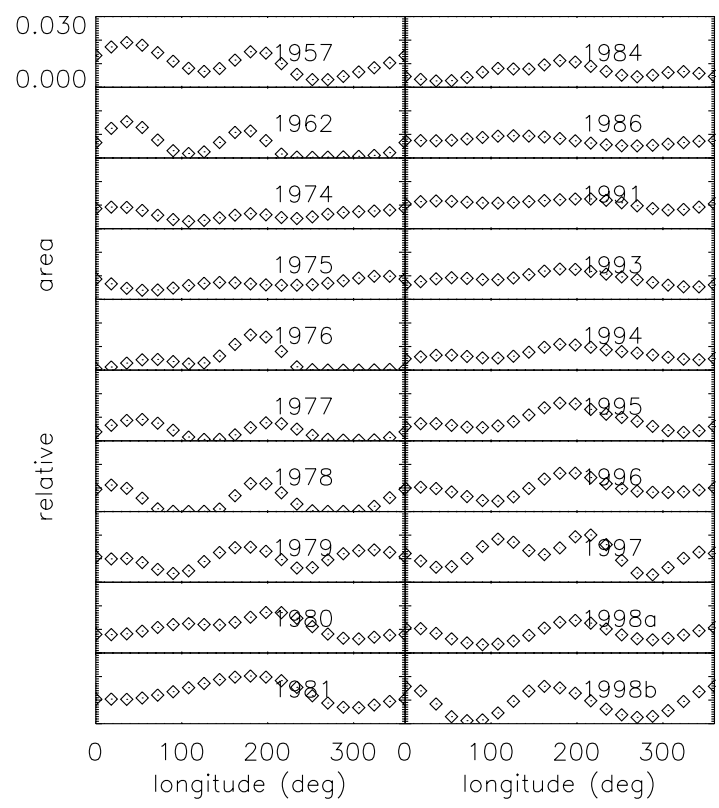

Fig. 13. The distribution of the relative spot area vs. longitude obtained from the T models for the K1 IV secondary. The area unit is the area of the star photosphere (see text).

observed around the substellar point in the secondary of AR Lac, although in that case there was some evidence for localized activity also at transition region and coronal levels (Lanza et al. 1998a).

Mean field dynamo models predict the possible existence of active or "preferred" longitudes related to nonaxisymmetric modes, when the amplitude of the stellar differential rotation does not exceed about half the solar value. Only non-axisymmetric field geometries with an azimuthal wavenumber $m=1$ have been found to be stable, 


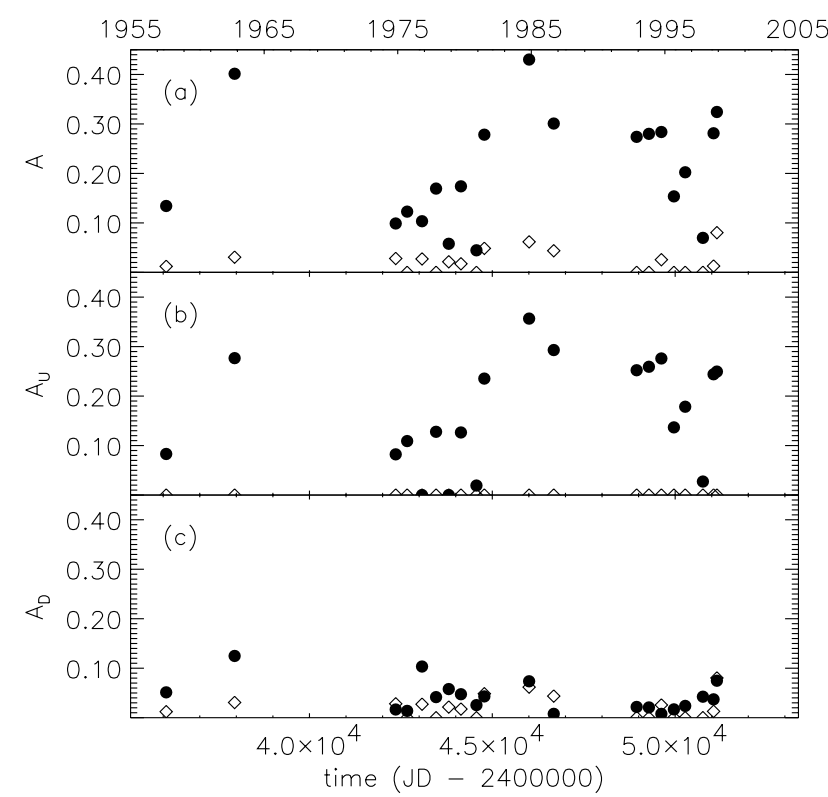

Fig. 14. The total spotted area $A$ a), the area of the uniform, evenly distributed spot component $A_{\mathrm{U}} \mathbf{b}$ ), and that of the discrete, longitudinally dependent spot component $A_{\mathrm{D}} \mathbf{c}$ ) vs. time for the F $8 \mathrm{~V}$ primary. The open symbols refer to the spotted areas derived from the ME models and the filled symbols to the spotted areas derived from the T models, respectively. The plotted areas are normalized to the photospheric area of the F8 V component.

but such a result may possibly be due to the many simplifying assumptions of present mean field models (Rädler et al. 1990; Moss et al. 1991; Rüdiger \& Elstner 1994; Moss et al. 1995). Moreover, it is possible that the binary nature of the RS CVn's has an influence on the activity of the single components (Schrijver \& Zwaan 1991). The mean field models of Moss \& Tuominen (1997) show that the magnetic field tends to concentrate along the line connecting the two components in close binaries, in general agreement with our findings for AR Lac and SZ Psc. Moreover, the presence of stationary preferential longitudes may be related to perturbations of the magnetic fields stored at the base of the convective envelopes, due to tidal forces, which eventually trigger the emergence of magnetic flux tubes (cf., e.g., Holzwarth \& Schüssler 2000).

Polar spots are not required by our solutions, but this leaves unanswered the question of their existence, claimed by several authors (see, however, Hatzes et al. 1996 and Hatzes 1998 for a critical discussion of the related spectroscopic evidence). The presence of large low-latitude spots, as deduced by our eclipse mapping, is a significant constraint for dynamo models. It suggests the existence of MGauss toroidal fields in the overshoot region below the convective envelope of the secondary component (Schüssler \& Solanki 1992; Schüssler et al. 1996; Granzer et al. 2000). Toroidal flux tubes with field strengths exceeding $(2-4) \times 10^{6} \mathrm{G}$ should have unstable azimuthal modes with azimuthal order $m=1$ or $m=2$, whose subsequent non-linear evolution can be quite complex

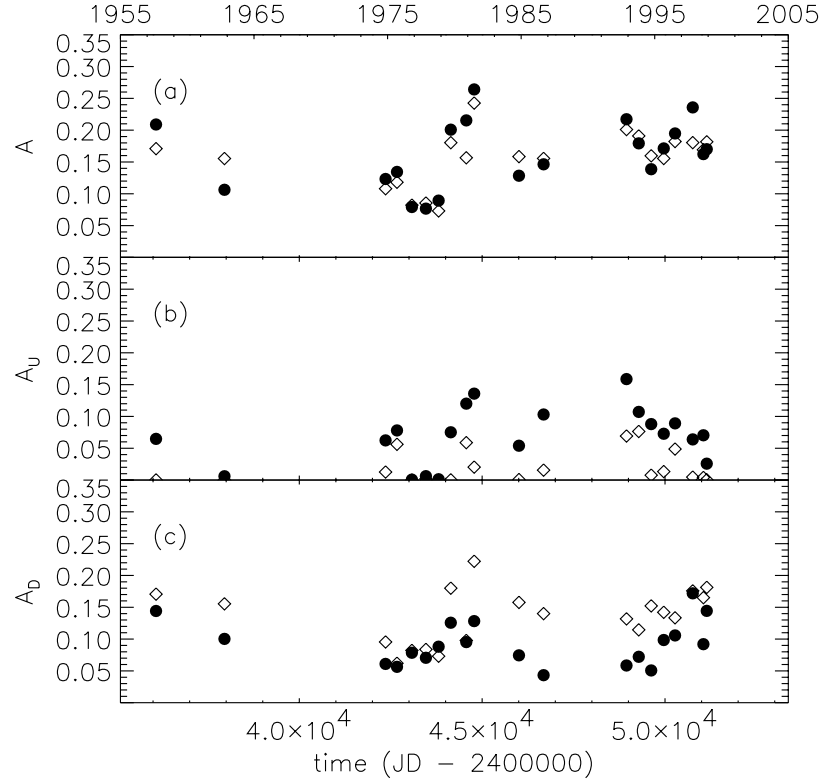

Fig. 15. The total spotted area $A$ a), the area of the uniform, evenly distributed spot component $A_{\mathrm{U}} \mathbf{b}$ ), and that of the discrete, longitudinally dependent spot component $A_{\mathrm{D}}$ c) vs. time for the K1 IV secondary. The open symbols refer to the spotted areas derived from the ME models and the filled symbols to the spotted areas derived from the T models, respectively. The plotted areas are normalized to the photospheric area of the K1 IV component.

(Caligari et al. 1995; Schüssler et al. 1996). Alternatively, a distributed dynamo operating in the bulk of the convective envelope might be invoked to explain low-latitude activity. In this scenario, the flux tubes might emerge near the equator in spite of the large Coriolis force because they are produced in layers not too far from the surface.

The time evolution of the spotted area on the K1 IV secondary component shows a possible short-term cycle with a period of about $13 \mathrm{yr}$ and possibly a longer term change with a period of approximately $30 \mathrm{yr}$. However, the time extension of the available data set is too short to draw definite conclusions because the present observations cover only one cycle of such modulation. Moreover, the star activity level seems to have been changing quite irregularly since 1991. Bakos \& Tremko (1987) modelled the out-of-eclipse light variations using a sinusoidal distortion wave and found that the amplitude of the wave changed with a period of $\sim 12 \mathrm{yr}$.

The observations of other RS CVn binaries show that the periods of the activity cycles are typically between one and two decades, so that a 13-yr cycle in the secondary component of SZ Psc compares well with the general behaviour of the class (Henry et al. 1995; Rodonò et al. 1995; Lanza et al. 1998a; Saar \& Brandenburg 1999; Oláh et al. 2000). We can use the decrease of the total spotted area observed between 1981 and 1984 to estimate the turbulent magnetic diffusivity $\eta_{\mathrm{t}}$ in the convective envelope of the K1 IV star, as done by, e.g., Lanza et al. (1998a) for the secondary of AR Lac, in the hypothesis that the variation 


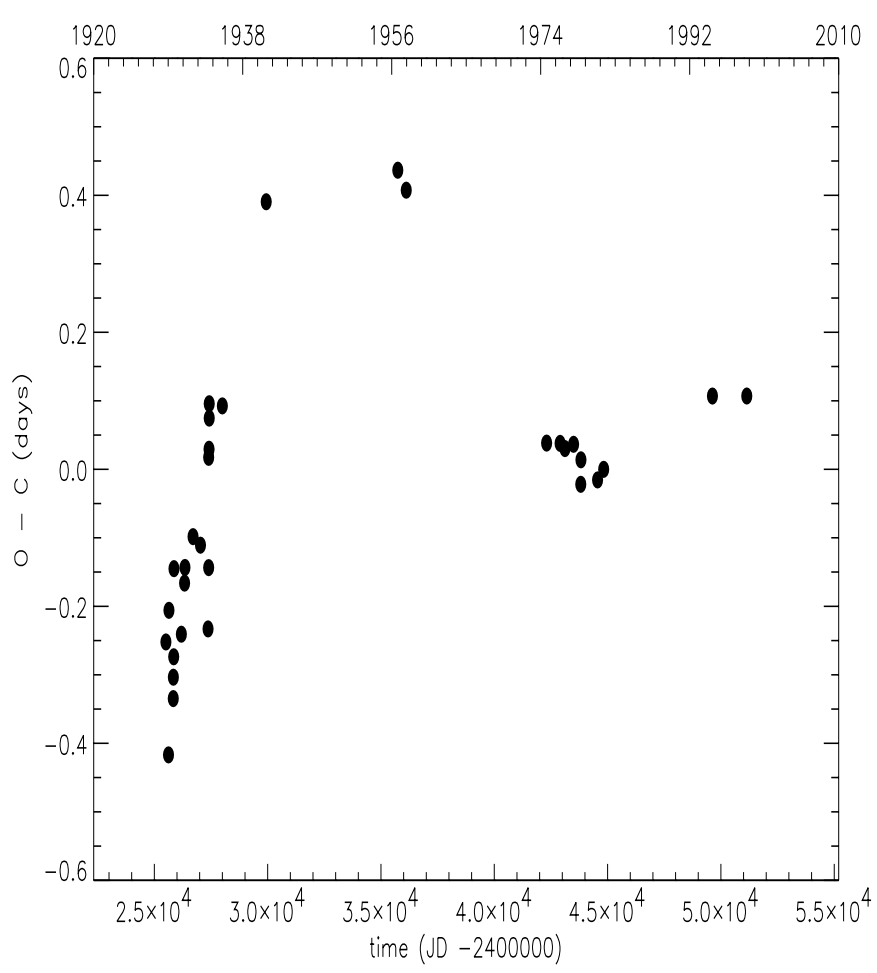

Fig. 16. Orbital period variations of SZ Psc vs. time. The $\mathrm{O}-\mathrm{C}$ residuals were computed adopting the ephemeris Min $I=$ $2444827.0047+3.9657889 \times E$.

is due only to the decay of the magnetic field. Adopting a radius of $5.1 R_{\odot}$ for the star (Popper 1988), and estimating $\eta_{\mathrm{t}} \approx \Delta A / \Delta t$, where $\Delta A$ is the decrease of the spot area in the time interval $\Delta t$, we find: $\eta_{\mathrm{t}} \sim 2.1 \times 10^{11}$ $\mathrm{m}^{2} \mathrm{~s}^{-1}$. It is similar to the value estimated for the K2 IV secondary of RS CVn (Rodonò et al. 1995). Such values are about 100 times larger than in the Sun, but they are not unreliable since on a $\mathrm{K}$ subgiant we expect that the mixing length and the velocity of the turbulent convection may be significantly larger than in the Sun (Schwarzschild 1975).

The spot activity of SZ Psc may also undergo longterm changes. Jakate et al. (1976) reported on old determinations of the depth of the primary minimum based on photographic observations. They indicated that its depth had decreased from $\approx 1.5 \mathrm{mag}$ to $0.67 \mathrm{mag}$ from $\sim 1930$ to 1974. This may correspond to a long-term decrease of the spot area on the secondary component, which is in view during primary minimum.

The variation of the orbital period in RS CVn binaries may be related to the magnetic activity of their secondary components, as suggested by Hall (1989). A viable physical mechanism to account for the observed changes was proposed by Applegate (1992), who suggested that the hydromagnetic dynamo may induce cyclic changes of the gravitational quadrupole moment of the secondary star. This mechanism can actually couple the orbital motion with magnetic activity. Lanza et al. (1998b) and Lanza \& Rodonò (1999b) developed further Applegate's model and made an extensive comparison with the observations. In

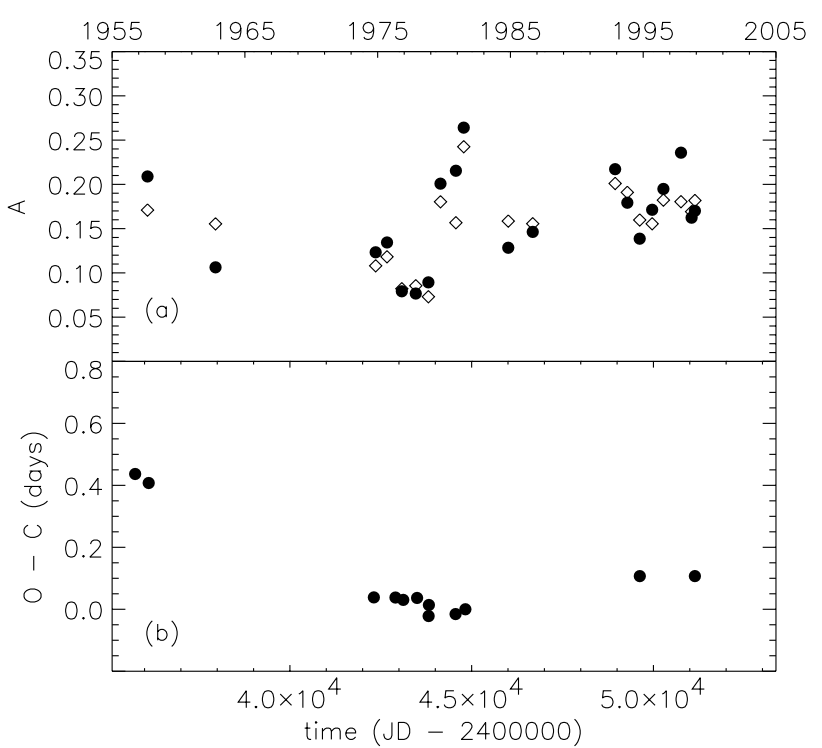

Fig. 17. The total spotted area on the K1IV component vs. time a) and the orbital period variations of SZ Psc vs. time b), plotted on the same time scale. The $\mathrm{O}-\mathrm{C}$ residuals were computed adopting the ephemeris $\operatorname{Min} I=2444827.0047+$ $3.9657889 \times E$.

particular, Lanza et al. (1998b) suggested that a possible mechanism for quadrupole moment changes may be a torsional oscillation maintained by a non-linear $\alpha^{2} \Omega$ dynamo. Such a model may account for the fact that the spot activity cycle has a length of about half that of the orbital period cycle, a phenomenon firstly described by Rodonò et al. (1995) in RS CVn. In the case of SZ Psc, there may be some hint for a non-sinusoidal spot cycle with a period of $\sim 30 \mathrm{yr}$, that is half of the $\sim 60 \mathrm{yr}$ orbital period cycle. A more extended sequence of data is needed to confirm such a suggestion.

In SZ Psc the more massive and larger K1 IV component has a gravitational quadrupole moment $\sim 500$ times that of the smaller F8 v primary and it is therefore the most suitable to support the Applegate's mechanism. In the period 1955-1981 the orbital period had a value smaller than in Eq. (4) ephemeris, because the $\mathrm{O}-\mathrm{C}$ plot showed a negative slope. In the 1981-1998 interval, it became almost equal to the period of the ephemeris, the $\mathrm{O}-\mathrm{C}$ staying approximately constant. The change of the orbital period apparently occurred between 1980 and 1990 and had an estimated amplitude of $\triangle P / P \sim 4 \times 10^{-5}$ (cf. Fig. 17). Such a variation is quite large for a RS CVn binary, but it is smaller than the change detected in HR 1099 which had a relative amplitude of $\triangle P / P \sim 10^{-4}$ (Donati 1999; Strassmeier \& Bartus 2000).

The model proposed by Lanza \& Rodonò (1999b) takes into account the effects of both centrifugal and magnetic forces on the figure of equilibrium of the secondary star and allowed them to relate the orbital period changes with the variation of the star magnetic and kinetic energies along the activity cycle. The increase of the orbital period of SZ Psc between 1980 and 1990 is accompanied by an 
increase of the mean area of the spots which may indicate an increase of the magnetic energy at the expenses of the kinetic energy. Such a variation is in qualitative agreement with the model proposed by Lanza \& Rodonò (1999b) in which the quadrupole moment decreases and the orbital period increases when the magnetic energy increases.

A quantitative comparison is also possible because the stellar parameters and the amplitude of the orbital period change are known. For the sake of simplicity, we assume that the magnetic field inside the secondary component is purely toroidal and that the sum of its kinetic energy of rotation and magnetic energy is constant during the variation of the quadrupole moment. A relative change of $\Delta P / P=4 \times 10^{-5}$ implies a change of the kinetic energy of rotation and of the magnetic energy of $\sim 1.9 \times 10^{37} \mathrm{~J}$, which leads to an overall change of the angular velocity of $\approx 1.9 \%$. Our analysis of the spot longitudes does not agree with such model prediction, because no evidence for a variable differential rotation was observed before and after the orbital period change. However, our spot modelling approach is more sensitive to the location of active longitudes than to invidual spot migration, because it is based on the analysis of seasonal light curves that tend to average out the photometric effects of the individual spot drift. Indeed the detection of differential rotation may become a difficult task when permanent active longitudes are present, as showed by, e.g., Eaton et al. (1996). The measurement of rotational modulation periods is a more suitable tool to look for rotation changes, even in the case of a complex active-longitude behaviour, as shown by, e.g., Rodonò et al. (2000). Unfortunately, photometric data are too sparse to apply such a technique to SZ Psc in the 1980-1990 period.

The corresponding change of the magnetic energy assumed in the model gives a mean field intensity of $\sim 1.6 \times 10^{5} \mathrm{G}$ in the convective envelope of the secondary. Such a high field is indeed strongly unstable to magnetic buoyancy, unless is it organized in the form of vertical flux tubes (cf. Lanza \& Rodonò 1999b). Unstable fields that buoyantly rise through the convective envelope and experience the effects of the Coriolis force, may produce a new kind of $\alpha$ effect that makes the stellar dynamo capable of working even when the conventional $\alpha$ effect is severely quenched (Brandenburg \& Saar 2000). Such a magnetically driven $\alpha$ effect might account for the strong fields that are required to produce the variation of the quadrupole moment and the low-latitude spot activity in the secondaries of RS CVn binaries.

It is interesting to compare our findings on the orbital period change with the previous study by Kalimeris et al. (1995). They discussed several physical mechanisms that might be responsible for the period variation and considered their advantages and drawbacks. Specifically, they showed that a light-time effect is an unlikely explanation for the orbital period change because the variation is not strictly periodic and there is no independent evidence for the required third body. In fact its mass would be so large to make it easily observable, unless it is a degenerate object. Therefore, they suggested that a combination of the Applegate's mechanism and an enhanced magnetic wind braking may explain both the cyclic change and the long-term trend of the orbital period variation.

\section{Conclusion}

The analysis of an extended sequence of light curves of SZ Psc led to several remarkable results. Our modelling approach based on Roche geometry and Kurucz's atmospheric models proved capable of fitting most of the light curves and provided us with maps of the spot covering factor we derived by the $\mathrm{ME}$ and $\mathrm{T}$ regularizing criteria. Moreover, taking into account the photometric effect of the spots, a consistent value of the $V$-band luminosity ratio between the two components was determined.

The light curve analysis gave us valuable information on the spot pattern showing, in particular, spots at low and intermediate latitudes and a persistent active region around the substellar point on the K1 IV secondary component. The eclipse mapping technique allowed us to estimate its extension that turned out to be of $\sim 40^{\circ}$ in several maps. Its detection also in the UV lines suggested that a solar-like active region was present there with chromospheric faculae overlying photospheric spots. Other active longitudes were detected on the K1 IV component, but their location and extension changed irregularly with time.

The spot pattern did not show evidence for a systematic drift produced by surface differential rotation and its changes were most likely dominated by the evolution of the individual active regions. The total spot area on the K1 IV star showed sizeable fluctuations and a possible short-term activity cycle with a period of $\sim 13 \mathrm{yr}$, particularly evident in the variation of the spot pattern unevenly distributed in longitude. Moreover, a long-term cycle with a period of $\approx 30 \mathrm{yr}$ could not be excluded.

Our single-band photometric data did not provide conclusive evidence for starspot activity on the F8 V primary, except for some hints of the presence of spots around the substellar point facing the secondary component.

The study of the orbital period variation suggested a possible modulation with a period of $\approx 60 \mathrm{yr}$ and showed that the most recent episode of period increase between 1980 and 1990 was accompanied by an increase of the mean spotted area on the K1IV secondary. Such observational results might be interpreted in the framework of the theoretical models recently proposed to relate orbital period changes and magnetic activity in close binaries. However, a more extended series of data is needed to derive more confident conclusions on the periodicity of the orbital period modulation and its connection with the spot area variation.

Acknowledgements. We wish to thank Dr. R. Kurucz for making available to us the atmospheric models for late-type stars used in this investigation and an anonymous Referee for a careful reading of the manuscript and useful comments. We acknowledge the extensive use of computing facilities at the 
Catania node of the Italian Astronet Network. This research has made use of the Simbad database, operated at the CDS, Strasbourg, France.

The acquisition of photometric data over so many years with the Catania APT has been possible thanks to the dedicated and highly competent technical assistance of a number of people, notably P. Bruno, E. Martinetti and S. Sardone. Research on stellar activity at Catania Astrophysical Observatory and at the Dept. of Physics and Astronomy of Catania University is funded by MURST (Ministero dell'Università e della Ricerca Scientifica e Tecnologica) and Regione Sicilia, whose financial support is gratefully acknowledged.

\section{References}

Allen, C. W. 1973, Astrophysical Quantities (The Athlone Press, London)

Antonopoulou, E., \& Williams, P. M. 1984, A\&A, 135, 61

Applegate, J. H. 1992, ApJ, 385, 621

Bakos, G. A., \& Tremko, J. 1987, BAICz, 38, 356

Blanco, C., Bodo, G., Catalano, S., et al. 1983, in Activity in Red-Dwarf Stars, ed. P. B. Byrne, \& M. Rodonò (D. Reidel Publ. Co., Dordrecht), 395

Brandenburg, A., \& Saar, S. H. 2000, in Stellar clusters and associations: convection, rotation and dynamos, ed. R. Pallavicini et al., ASP Conf. Ser., 198, 381

Caligari, P., Moreno-Insertis, F., \& Schüssler, M. 1995, ApJ, 441,886

Cameron, A. C., \& Hilditch, R. W. 1997, MNRAS, 287, 567

Dempsey, R. C., Linsky, J. L., Fleming, T. A., \& Schmitt, J. H. M. M. 1993, ApJS, 86, 599

Diaz-Cordoves, J., Claret, A., \& Gimenez, A. 1995, A\&AS, 110,329

Donati, J.-F. 1999, MNRAS, 302, 457

Doyle, J. G., Mitrou, C. K., Mathioudakis, M., \& Antonopoulou, E. 1994b, A\&A, 283, 522

Doyle, J. G., Mitrou, C. K., Mathioudakis, M., et al. 1994a, A\&A, 291, 135

Eaton, J. A. 1977, IBVS, 1297, 1

Eaton, J. A. 1980, IAU Comm. 27, Archive for Unpublished Obs. of Variable Stars, file (121)

Eaton, J. A. 1992, in Surface Inhomogeneities in Late-Type Stars, ed. P. B. Byrne, \& D. J. Mullan, LNP 397 (SpringerVerlag, Berlin), 15

Eaton, J. A., \& Hall, D. S. 1979, ApJ, 227, 907

Eaton, J. A., Henry, G. W., Bell, C., \& Okorogu, A. 1993, AJ, 106,1181

Eaton, J. A., Henry, G. W., \& Fekel, F. C. 1996, ApJ, 462, 888

Eaton, J. A., Scaltriti, F., Cerruti-Sola, M., et al. 1982, Ap\&SS, 82,289

Fernandez-Figueroa, M. J., Montes, D., De Castro, E., \& Cornide, M. 1994, ApJS, 90, 433

Florkowski, D. R., Johnston, K. J., Wade, C. M., \& de Vegt, C. 1985, AJ, 90, 2381

Frasca, A., \& Catalano, S. 1994, A\&A, 284, 883

Granzer, Th., Schüssler, M., Caligari, P., \& Strassmeier, K. G. 2000, A\&A, 355, 1087

Gray, D. F. 1992, the Observation and Analysis of Stellar Photospheres (Cambridge Univ. Press, Cambridge)

Guinan, E. F., \& Gimenez, A., 1993, in The Realm of Interacting Binary Stars, ed. J. Sahade, G. E. McCluskey, Jr., \& Y. Kondo (Kluwer Ac. Publ.), 51
Gull, S. F., \& Skilling, J. 1984, in Indirect Imaging, ed. J. A. Roberts (Cambridge Univ. Press, Cambridge), 267

Hall, D. S. 1976, in Multiple Period Variable Stars, ed. W. S. Fitch, IAU Coll., 29, 287

Hall, D. S. 1989, Space Sci. Rev., 50, 219

Hall, D. S., \& Kreiner, J. M. 1980, AA, 30, 388

Hatzes, A. 1998, A\&A, 330, 541

Hatzes, H. A., Vogt, S. S., Ramseyer, T. F., \& Misch, A. 1996, ApJ, 469, 808

Henry, G. W., Eaton, J. A., Hamer, J., \& Hall, D. S. 1995, ApJS, 97, 513

Holzwarth, V., \& Schüssler, M. 2000, AN, 321, 175

Horne, J. H., \& Baliunas, S. L. 1986, ApJ, 302, 757

Huenemoerder, D. P., \& Ramsey, L. W. 1984, AJ, 89, 549

Jakate, S. 1979, IBVS, 1578, 1

Jakate, S., Bakos, G. A., Fernie, J. D., \& Heard, J. F. 1976, AJ, 81, 250

Kalimeris, A., Mitrou, C. K., Doyle, J. G., Antonopoulou, E., \& Rovithis-Livaniou, H. 1995, A\&A, 293, 371

Kang, Y. W., \& Wilson, R. E. 1989, AJ, 97, 848

Kopal, Z. 1989, The Roche Problem (Kluwer Ac. Publ., Dordrecht)

Kurucz, R. 2000, http://cfaku5.harvard.edu

Lampton, M., Margon, B., \& Bowyer, S. 1976, ApJ, 208, 177

Lanza, A. F., Catalano, S., Cutispoto, G., Pagano, I., \& Rodonò, M. 1998a, A\&A, 332, 541

Lanza, A. F., \& Rodonò, M. 1999a, in Solar and stellar activity: similarities and differences, ed. C. J. Butler, \& J. G. Doyle, ASP Conf. Ser., 158, 121

Lanza, A. F., \& Rodonò, M. 1999b, A\&A, 349, 887

Lanza, A. F., Rodonò, M., \& Rosner, R. 1998b, MNRAS, 296. 893

Maltby, P. 1992, in Sunspots: Theory and Observations, ed. J. H. Thomas, \& N. O. Weiss (Kluwer Ac. Publ., Dordrecht), 103

Marino, G. 1999, Ph.D. Thesis, Univ. of Catania

Messina, S., Guinan, E. F., Lanza, A. F., \& Ambruster, C. 1999, A\&A, 347, 249

Mitrou, C. K., Mathioudakis, M., Doyle, J. G., \& Antonopoulou, E. 1997, A\&A, 317, 776

Moss, D., Tuominen, I., \& Brandenburg, A. 1991, A\&A, 245, 129

Moss, D., Barker, D. M., Brandenburg, A., \& Tuominen, I. 1995, A\&A, 294, 155

Moss, D., \& Tuominen, I. 1997, A\&A, 321, 151

Narayan, R., \& Nityananda, R. 1986, ARA\&A, 24, 127

Okazaki, A. 1979, IBVS, 1560, 1

Oláh, K., Kolláth, Z., \& Strassmeier, K. G. 2000, A\&A, 356, 643

Oláh, K., Kövari, Zs., Bartus, J., et al. 1997, A\&A, 321, 811

Percy, J. R. 1984, JRASC, 79, 113

Percy, J. R., \& Welch, D. L. 1982, JRASC, 76, 185

Piskunov, N. E., Tuominen, I., \& Vilhu, O. 1990, A\&A, 230, 363

Popper, D. M. 1988, AJ, 96, 1040

Pye, J. P., McGale, P. A., Allan, D. J., et al. 1995, MNRAS, 274,1165

Rädler, K.-H., Wiedemann, E., Brandenburg, A., Meinel, R., \& Tuominen, I. 1990, A\&A, 239, 413

Rodonò, M., \& Lanza, A. F. 1996, in Magnetodynamic Phenomena in the Solar Atmosphere, Prototypes of Stellar Magnetic Activity, ed. Y. Uchida, T. Kosugi, \& H. S. Hudson (Kluwer Ac. Publ., Dordrecht), 375 
Rodonò, M., Lanza, A. F., \& Catalano, S. 1995, A\&A, 301, 75

Rodonò, M., Lanza, A. F., \& Becciani, U. 2001, A\&A, 371, 174

Rodonò, M., Messina, S., Lanza, A. F., Cutispoto, G., \& Teriaca, L. 2000, A\&A, 358, 624

Rüdiger, G., \& Elstner, D. 1994, A\&A, 281, 46

Saar, S. H., \& Brandenburg, A. 1999, ApJ, 524, 295

Scargle, J. D. 1982, ApJ, 263, 835

Schrijver, C. J., \& Zwaan, C. 1991, A\&A, 251, 183

Schüssler, M., \& Solanki, S. K. 1992, A\&A, 264, L13

Schüssler, M., Caligari, P., Ferriz-Mas, A., Solanki, S. K., \& Stix, M. 1996, A\&A, 314, 503

Schwarzschild, M. 1975, ApJ, 195, 137
Spruit, H. C. 1982, A\&A, 108, 348

Spruit, H. C., \& Weiss, A. 1986, A\&A, 166, 167

Strassmeier, K. G., \& Bartus, J. 2000, A\&A, 354, 537

Strassmeier, K. G., Hall, D. S., Fekel, F. C., \& Scheck, M. 1993, A\&AS, 100, 173

Thompson, K. 1987, IBVS, 3119, 1

Tumer, O., Kurutac, M., Tunca, Z., et al. 1980, IBVS, 1741, 1

Tunca, Z. 1984, Ap\&SS, 105, 23

Vincent, A., Piskunov, N. E., \& Tuominen, I. 1993, A\&A, 278, 523

Vogt, S. S., Penrod, G. D., \& Hatzes, A. P. 1987, ApJ, 321, 496 